Pacific Journal of Mathematics

UNIVERSAL DERIVATIONS AND UNIVERSAL RING 


\title{
UNIVERSAL DERIVATIONS AND UNIVERSAL RING CONSTRUCTIONS
}

\author{
George M. Bergman and Warren Dicks
}

If a $K$-ring $S$ is constructed from a $K$-ring $R$ by adjoining certain new generators and relations, then the $S$-bimodule $\Omega_{K}(S)$ with a universal $K$-derivation $d: S \rightarrow \Omega_{K}(S)$ can be constructed from the corresponding $R$-bimodule $\Omega_{K}(R)$ by extending scalars to $S$, and adjoining formal derivatives of the new generators and relations. By studying this bimodule it is shown that a large number of natural universal constructions preserve the class of right hereditary $K$-rings ( $K$ semisimple Artinian), including the constructions of universal localization (which had resisted earlier techniques) and certain direct limits of known constructions. The same technique gives information on Euler characteristics of modules (Lewin-Schreier formulas). To study universal localizations of a ring $R$ which may not contain a semisimple Artin ring $K$, a different technique is used.

Let $R$ be a ring (associative, with 1 ) and $f: P \rightarrow Q$ a homomorphism of finitely generated projective right $R$-modules. Then there exists an $R$-ring $S=R\left\langle f^{-1}\right\rangle$ having a map of projectives, $f^{-1}: Q \otimes_{R} S \rightarrow P \otimes_{R} S$ inverse to $f$ (more precisely, inverse to $f \otimes_{R} S$ ), and such that $S$ and $f^{-1}$ are universal for this property. This construction generalizes that of adjoining to $R$ a universal inverse of an element $f \in R$. We may simultaneously adjoin such inverses to a whole family of maps. We shall call an $R$-ring $S$ so obtained a universal (2-sided) localization of $R$. The main result of this paper-of which we shall prove two versions by two different methods-is:

(0) A universal localization $S$ of a right hereditary ring $R$ is right hereditary.

Universal localization was one of a large class of constructions examined in [2] and [3], including also the formation of the coproduct $S$ of a family of rings $R_{\alpha}$, the universal adjunction to a ring $R$ of an isomorphism between two given finitely generated projective $R$-modules $P$ and $Q$, and the adjunction to $R$ of a universal idempotent endomorphism of a finitely generated projective module $P$. For these last three and many others, it was proved that when the construction is performed "over" a semisimple Artinian base ring $K$, a large part of the module theory of the constructed 
ring $S$ reduces to that of the given rings. In particular, if the given rings have right global dimension $\leqq n$ for some positive $n$, then so does $S$.

However universal localization, and the similar constructions of universal right and left localization (adjoining 1-sided inverses to elements or maps) were stubbornly resistant to the techniques used there.

The first of the proofs of (0) that we give here, will yield the result "if the given rings are right hereditary then the constructed ring is right hereditary" not only for 2-sided localization, but also for universal right and left localization, for all the constructions treated in [2] and [3], and for a large class of direct limits of such constructions. But like the methods of [2] and [3], this approach requires the assumption of a semisimple Artinian base ring $K$, over which the constructions are performed. Indeed, most of these constructions are sensitive to base ring, and fail to have such good properties when performed over other sorts of base ring, such as $Z$. Cf. [18], and [2] Example 12.1. For another example, if $k$ is a field, the coproduct of two copies of the polynomial ring $k[t]$ over the common subring $k\left[t^{2}\right]$ will, like the example from [2] just cited, have global dimension 2 , though their coproduct over $k$, a free associative algebra, is hereditary.

However, for reasons of general nonsense (see last paragraph of $\$ 2.9$ below) the construction of universal 2 -sided localization is independent of base ring. Our second proof of (0) will establish (0) for this construction only, but without reference to any subring $K$.

Let us now sketch the approach of the first proof. If $K \rightarrow R$ is any ring homomorphism, there exists an $R$-bimodule $\Omega_{K}(R)$ with a universal $K$-derivation $d: R \rightarrow \Omega_{K}(R)$ (cf. [6]). This fits into a short exact sequence (recalled below, (61)) which is intimately connected with the module theory of $R$. In particular, when $K$ is semisimple Artinian there is a simple criterion in terms of $\Omega_{K}(R)$ for $R$ to be right hereditary (Proposition 3.1). But for all the universal constructions in question, the bimodule $\Omega_{K}(S)$ associated with the constructed ring $S$ can be neatly described in terms of the corresponding bimodules for the given rings (shown in $\S 2$ ). It will follow that if the given rings are right hereditary, so is the constructed ring (Theorem 3.3).

This method does not yield the detailed information on the category of $S$-modules that was obtained in [2] and [3] for most of these constructions. However, the conclusions that if the given rings $R_{\alpha}$ are semihereditary or of global dimension $\leqq n$ (for $n>0$ ) then $S$ will also be so can again be obtained if we assume $S$ is flat over the $R_{\alpha}$. In fact, the constructions treated in [2] and [3] 
always do yield flat extensions; but universal localization may not! We give examples in $\S 4$ of a semihereditary ring (in fact, a semifir) having a universal localization that is not semihereditary (nor even 1-hereditary), and of a ring of global dimension 2 which has a universal localization of global dimension $\infty$.

A nice feature of this approach is that it is fairly easy to keep track of what happens when one goes to a direct limit of constructions, because $\Omega_{K}$ respects colimits. As an application, we shall be able to strengthen a result of [3] to conclude that any abelian semigroup satisfying some obvious necessary conditions can occur as the full semigroup (under $\oplus$ ) of isomorphism classes of finitely generated projective right modules over a hereditary ring (Theorem 3.4 and paragraph following).

We shall also generalize some results of J. Lewin [20] on Euler characteristics of modules (§3.8).

We now sketch our other method of proving (0), this time without a base ring. One knows that if $R \rightarrow S$ is a flat ring epimorphism, then one easily obtains r.gl.dim. $S \leqq$ r.gl.dim. $R$. We shall show that if one only wants this inequality for the case r.gl.dim. $R=1$, then the condition " $S$ is flat" can be weakened to $\operatorname{Tor}_{1}^{R}(S, S)=0$. We then prove that the latter equation holds for all universal localizations. To do so we borrow a trick from [2], [3]: Verify the result in a "generic" case, and reduce the general case to this one via pushouts and matrix rings. The hardest work is in showing that our generalization of flatness (unlike flatness itself) respects pushouts of rings.

1. Conventions and general observations. All rings will be associative with 1 .

Let us fix a ring $K$. Then a $K$-ring will mean a ring $R$ given with a homomorphism $K \rightarrow R$. Homomorphisms of $K$-rings are ring homomorphisms $R \rightarrow R^{\prime}$ forming commuting triangles with the given maps from $K$.

If $B$ is a bimodule over a $K$-ring $R$, then a $K$-derivation from $R$ to $B$ will mean a homomorphism of abelian groups, $\delta: R \rightarrow B$, which satisfies

$$
\begin{aligned}
\delta(x y) & =(\delta x) y+x(\delta y) & & (x, y \in R) \\
\delta \alpha & =0 & & (\alpha \in K) .
\end{aligned}
$$

Let $(R, B)$ denote $R \oplus B$ made a $K$-ring using component-wise addition, and with multiplication and $K$-structure given respectively by

$$
\begin{aligned}
(r+b)\left(r^{\prime}+b^{\prime}\right) & =r r^{\prime}+\left(r b^{\prime}+b r^{\prime}\right) & & \left(r, r^{\prime} \in R ; b, b^{\prime} \in B\right) \\
\alpha_{(R, B)} & =\alpha_{R}+0_{B} & & (\alpha \in K) .
\end{aligned}
$$


Then a $K$-derivation $R \rightarrow B$ can be characterized as a map $\delta$ such that the map $r \mapsto r+\delta r$ is a homomorphism of $K$-rings $R \rightarrow(R, B)$. (Compare the characterization in [6], Introduction.)

Given a $K$-ring $R$, there exists by general nonsense an $R$-bimodule $\Omega_{K}(R)$ with a universal $K$-derivation $d: R \rightarrow \Omega_{K}(R)$. (This is the $R=S=T$ case of the $(S, T)$-bimodule called $\Omega^{(K, R)}(S, T)$ in [6].) If $R$ is presented as a $K$-ring by a set of generators $X$ and a set of relations $Z$, then $\Omega_{K}(R)$ will be presented as $R$-bimodule by a set of generators $\{d x \mid x \in X\}$ and the relations obtained by formally differentiating the equations of $Z$. (E.g., if $R$ is given by two generators $x, y$, and one relation $x \alpha y=\beta$ for some $\alpha, \beta \in K$, then the $R$-bimodule $\Omega_{K}(R)$ will be presented by generators $d x$ and $d y$ and the one relation $(d x) \alpha y+x \alpha(d y)=0$.) Hence, if $S$ is a $K$-ring obtained by adjoining to $R$ additional generators $X^{\prime}$ and additional relations $Z^{\prime}, \Omega_{K}(S)$ may be obtained from $\Omega_{K}(R)$ by first tensoring on both sides with $S$ to get an $S$-bimodule, then adjoining the corresponding new generators and relations.

If $P$ is a right module over a ring $R$, and $S$ is an $R$-ring, then the $S$-module $P \otimes_{R} S$ will, when there is no danger of ambiguity, frequently be abbreviated $P^{\otimes}$. Likewise if $P$ is a left module we will write ${ }^{\otimes} P$ for $S \otimes P$ and when $B$ is a bimodule, ${ }^{\otimes} B^{\otimes}$ for $S \otimes B \otimes S$. (Aside from the advantage of brevity, this will have the syntactic value of allowing us to refer to $P^{\otimes}$ while $S$ is being defined.)

The ring of $n \times n$ matrices over $R$ will be denoted $M_{n}(R)$. If $B$ is an $R$-bimodule (possibly $R$ itself), then ${ }^{m} B^{n}$ will denote the set of all $m \times n$ matrices with entries in $B$, which is in a natural way an $\left(M_{m}(R), M_{n}(R)\right.$ )-bimodule; equivalently, a right $M_{n}(R)$-module on which $M_{m}(R)$ acts on the left by endomorphisms, or a left $M_{m}(R)$ module on which $M_{n}(R)$ acts on the right. When one of $m$ or $n$ is 1 , this superscript will be omitted, so for instance, ${ }^{m} R$ denotes the free right $R$-module of rank $m$, represented by column vectors of height $m$, and its endomorphisms are represented by members of $M_{m}(R)$ acting on the left. More generally, of course, we can multiply nonsquare matrices of appropriate sorts, e.g., ${ }^{m} R^{n} \times{ }^{n} B^{r} \rightarrow$ ${ }^{m} B^{r}$, and also form rows or columns of elements of 1-sided $R$-modules.

Added in proof. If $A \in{ }^{m} R^{n}$, the image of ${ }^{n} R$ under $A$ will be written $A^{n} R$. We did not realize that a superscript cannot be brought close to the left of an italic letter. So the reader must keep in mind that expressions like $A^{n} R$ should be read as $A\left({ }^{n} R\right)$, not as $\left(A^{n}\right) R$. Likewise, $R^{m} A$ means $\left(R^{m}\right) A$, the image of $R^{m}$ under the right action of $A$.) 
If $A=\left(\left(a_{i j}\right)\right) \in{ }^{m} R^{n}$ and $\delta: R \rightarrow B$ is a $K$-derivation, we shall write $\delta A$ for the matrix $\left(\left(\delta a_{i j}\right)\right) \in{ }^{m} B^{n}$. It is easy to check that for matrices as for elements:

$$
\begin{aligned}
\delta\left(A+A^{\prime}\right) & =\delta A+\delta A^{\prime} \\
\left(\delta A A^{\prime}\right) & =A\left(\delta A^{\prime}\right)+(\delta A) A^{\prime} \\
\delta A & =0 \text { if } A \in{ }^{m} K^{n} .
\end{aligned}
$$

Note that if $\delta A=0$, it does not follow that $\delta A^{\prime}=0$ for any similar matrix $A^{\prime}=P A P^{-1}$. (The reader may obtain the correct formula relating $\delta A^{\prime}$ and $\delta A$ by differentiating the relation $P A=A^{\prime} P$.) Hence we cannot speak of "applying $\delta$ to a module homomorphism", but only to a matrix representing a homomorphism of free modules, written in terms of specified bases.

2. The behavior of $\Omega_{K}(R)$ under universal constructions.

Construction 2.1. Universal 2-sided localization. In the "classical" form of this construction one takes a subset $\Theta$ of the given ring $R$, adjoins a new generator $u_{a}$ to $R$ for each $a \in \Theta$, and imposes relations $u_{a} a=a u_{a}=1$, getting a ring $\left.R<a^{-1}(a \in \Theta)\right\rangle$ or briefly, $R\left\langle\Theta^{-1}\right\rangle$. The more general case where $\Theta$ consists of matrices over $R$ (not even necessarily square) offers no essential complications: if $A \in \Theta$ is an $m \times n$ matrix, we introduce an $n \times m$ matrix $U_{A}$ of new indeterminates and the appropriate matrix relations.

Inverting a morphism of finitely generated projective modules, $a: P \rightarrow Q$ is a bit more complicated. We first choose representations of $P$ and $Q$ as direct summands of free modules

$$
\begin{array}{llll}
P=E^{m} R & \text { where } & E^{2}=E, & E \in M_{m}(R), \\
Q=F^{n} R & \text { where } & F^{2}=F, & F \in M_{n}(R),
\end{array}
$$

say with complements $P^{\prime}=(I-E)^{m} R, Q^{\prime}=(I-F)^{n} R$. Then $a$ can be represented by a homomorphism ${ }^{m} R \rightarrow{ }^{n} R$ which kills $P^{\prime}$ and has image in $Q$, i.e., by an $n \times m$ matrix $A$ satisfying

$$
A E=A=F A \text {. }
$$

A map from $Q$ to $P$ inverse to $a$ would be represented by an $m \times n$ matrix $U$ satisfying

$$
\begin{gathered}
E U=U=U F \\
U A=E, \quad A U=F .
\end{gathered}
$$

So to introduce such an inverse universally, we adjoin $\mathrm{nm}$ indeterminates $u_{i j}$ and impose the relations (3). (Cf. [3], §3.) The 
matrix $U$ over the resulting ring $S$ will determine an inverse $u$ to the map $a^{\otimes}: P^{\otimes} \rightarrow Q^{\otimes}$. Though our construction was based on the nonunique choice of the identification (1), the result is unique up to canonical isomorphism because of its universal property. Likewise, generally, given a family $\Theta$ of morphisms $a: P_{a} \rightarrow Q_{a}$ of finitely generated projective right $R$-modules, we get a ring

$$
S=R\left\langle\Theta^{-1}\right\rangle=R\left\langle a^{-1}: Q_{a}^{\otimes} \longrightarrow P_{a}^{\otimes}(a \in \Theta)\right\rangle .
$$

We shall now show

(4) If $R$ is a $K$-ring and $S=R\left\langle\Theta^{-1}\right\rangle$ a universal 2-sided localization, then $\Omega_{K}(S)={ }^{\otimes} \Omega_{K}(R)^{\otimes}$ (that is, the $S$-bimodule $\left.S \otimes_{R} \Omega_{K}(R) \otimes_{R} S\right)$.

We shall do this by showing that $\otimes_{\Omega_{K}}(R)^{\otimes}$ has the correct universal property. First, to obtain a derivation $d_{S}: S \rightarrow{ }^{\otimes} \Omega_{K}(R)^{\otimes}$, consider the ring homomorphisms

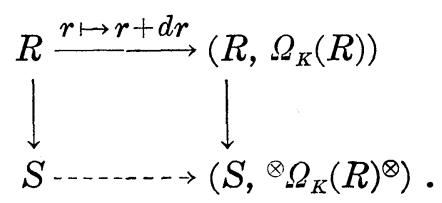

By the universal property of $S=R\left\langle\Theta^{-1}\right\rangle$ there will exist a homomorphism making the diagram commute if and only if each of the $R$-module homomorphisms $a: P_{a} \rightarrow Q_{a}$ in $\Theta$ becomes invertible on extending scalars to the ring $\left(S,{ }^{\otimes} \Omega_{K}(R)^{\otimes}\right)$. But note that ${ }^{\otimes} \Omega_{K}(R)^{\otimes}$ is a nilpotent 2-sided ideal of $\left(S,{ }^{\otimes} \Omega_{K}(R)^{\otimes}\right)$, and by construction of $S$, the given maps are invertible modulo this ideal. Inverses of morphisms of projective modules lift modulo a nilpotent ideal ([1], Prop. III.2.12(a), p. 90), hence the desired ring map exists, and its second component will be the desired derivation.

The universal property of this derivation is shown similarly. Given a $K$-derivation $\delta: S \rightarrow B$ where $B$ is an $S$-bimodule, we first use the universal property of $\Omega_{K}(R)$ to factor $\delta \mid R$ through $\Omega_{K}(R)$, then the universal property of extension of scalars to factor the resulting map through an $S$-bimodule homomorphism (dotted arrow in (6)):

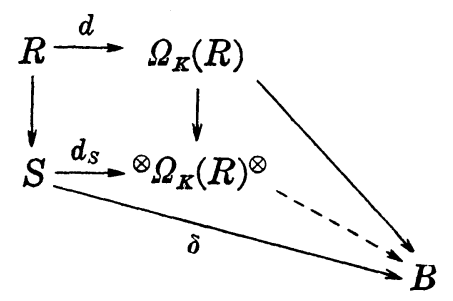


This makes the upper triangle of (6) commute. We need commutativity of the lower triangle; to get this recall that $R \rightarrow S$ is an epimorphism in the category of rings, i.e., any two ring maps $S \rightrightarrows T$ agreeing on $R$ must be equal. (This is because the image of $S$ under any ring homomorphism is generated over the image of $R$ by the components of 2-sided inverses of module-maps, and 2-sided inverses are always unique.) Applying this to maps $S \rightarrow(S, B)$ we see that any two derivations $S \rightarrow B$ agreeing on $R$ are equal. This proves the required commutativity and completes the proof of (4).

Let us now find explicit formulae for the action of $d$ on the generators we have introduced.

If $\Theta$ consists merely of elements of $R$, we can apply $d$ to the equations $a a^{-1}=1, a^{-1} a=1$, getting

$$
(d a) a^{-1}+a\left(d a^{-1}\right)=0, \quad\left(d a^{-1}\right) a+a^{-1}(d a)=0,
$$

either of which is equivalent to

$$
d a^{-1}=-a^{-1}(d a) a^{-1} .
$$

Again the matrix case is essentially the same. For the general case, let us apply $d$ to the last equation of (3), getting $A(d U)+$ $(d A) U=d F$, then left-multiply by $U$, getting $E(d U)+U(d A) U=U(d F)$. If we now apply $d$ to the equation $U=E U$ to get $d U=E(d U)+$ $(d E) U$, and eliminate $E(d U)$ between this and the preceding equation, we get

$$
d U=U(d F)-U(d A) U+(d E) U
$$

the desired formula. (Cf. (8).)

Equation (9) (or in the "easy" case, (8)) shows how when one goes from $R$ to $S$, the new relations introduced in $\Omega_{K}$ reduce the new generators to $S$-bimodule expressions in the old generators. Now, one can also verify a converse result, that (9) implies all the relations obtained by applying $d$ to (3) (assuming (2) and the result of applying $d$ to it as given). Hence the new relations in $\Omega_{K}(S) d o$ nothing but reduce the new generators to expressions in the old elements, and this yields an alternative proof of (4). However, the verification alluded to, though immediate when $\Theta \subseteq R$, where it is just the observation that $(8) \Leftrightarrow(7)$, is a rather opaque computation in the general case, which is why we preferred to give a conceptual proof of (4).

Having the above description of $\Omega_{K}(S)$, we could now prove that if $R$ is right hereditary, so is $S$. (The reader who wishes may turn directly to $\S 3$ for this proof.) However, we wish to get similar results for other universal constructions, and for those such 
as 1-sided localization which depend nontrivially on the base ring, the development of a corresponding description of $\Omega_{K}(S)$ will take more work. Subsections $2.3 \mathrm{ff}$. will be devoted to this development.

First, however, let us note a natural "appendage" to the construction just considered. It was noted in [3] that the construction of universally annihilating a finitely generated projective module $P=E^{m} R$, i.e., dividing out by the idempotent ideal generated by the entries of $E$, was a special case of universal localization, equivalent to universally inverting the zero endomorphism of $P$, or the $m \times m$ matrix $I-E$. Hence (4) applies to this construction. But there is a more general result:

Construction 2.2. Dividing out by an idempotent ideal.

$$
\begin{aligned}
& \text { If } R \text { is a } K \text {-ring and } S=R / I \text {, where } I \text { is an ideal of } R \text { with } \\
& I^{2}=I \text {, then } \Omega_{K}(S)={ }^{\otimes} \Omega_{K}(R)^{\otimes} \text {. }
\end{aligned}
$$

For we know that $\Omega_{K}(S)$ may be obtained from $\Omega_{K}(R)$ by tensoring on both sides with $S$ and imposing the relations

$$
d I=0 \text {. }
$$

But from the hypothesis $I=I^{2}$ we see that in $\Omega_{K}(R)$

$$
d I \subseteq(d I) I+I(d I) \subseteq \Omega_{K}(R) I+I \Omega_{K}(R)
$$

so the relations (11) become vacuous when we tensor $\Omega_{K}(R)$ with $S=R / I$; so $\Omega_{K}(S)$ is described by $(10)$.

((4) and (10) should not lead one to guess that $\Omega_{K}(S)={ }^{\otimes} \Omega_{K}(R)^{\otimes}$ for all ring epimorphisms $R \rightarrow S$. In fact a ring map $R \rightarrow S$ is an epimorphism if and only if the natural map ${ }^{\otimes} \Omega_{K}(R)^{\otimes} \rightarrow \Omega_{K}(S)$ is surjective; this can be deduced from [6], Theorem 18, putting our present $R, S$ for $K, R$, and ignoring our " $K$ ". Examples where this map is not injective are given by any factor-map $R \rightarrow R / I$ where $I$ is a nonidempotent ideal and $K$ is, say, a field. This can be deduced from [6] Theorem 3, taking $\mathfrak{a}=\mathfrak{b}=I$; cf. (97) below.)

Construction 2.3. Coproducts of K-rings. Let $\left(R_{\alpha}\right)_{\alpha \in A}$ be a family of $K$-rings, and $S=\Perp_{A} R_{\alpha}$ its coproduct in the category of $K$-rings. (In old language its "free product amalgamating $K$ ".)

$S$ may be presented as a $K$-ring by the disjoint union of families of generators and relations for the separate $R_{\alpha}$ 's. Hence $\Omega_{K}(S)$ is presented as an $S$-bimodule by the disjoint union of the same systems of generators and relations that arise in presenting the $\Omega_{K}\left(R_{\alpha}\right)$ as $R_{\alpha}$-bimodules. This yields

$$
\text { If } S=\Perp_{A} R_{\alpha} \text {, then } \Omega_{K}(S)=\bigoplus_{A}{ }^{\otimes} \Omega_{K}\left(R_{\alpha}\right)^{\otimes} \text {. }
$$


An immediate generalization of this argument gives:

Construction 2.4. Colimits of K-rings. Let $\mathscr{R}$ be a functor from a small category $A$ to the category of $K$-rings, and $S$ its colimit. For every object $\alpha \in O b(A)$ we form the $\mathscr{R}(\alpha)$-bimodule $\Omega_{K}(\mathscr{R}(\alpha)$ ), and for every morphism $\alpha \rightarrow \beta$ in $A$, we get a morphism of $\mathscr{R}(\alpha)$-bimodules, $\Omega_{K}(\mathscr{R}(\alpha)) \rightarrow \Omega_{K}(\mathscr{R}(\beta))$, which on tensoring with $S$ gives a morphism of $S$-bimodules ${ }^{\otimes} \Omega_{K}(\mathscr{R}(\alpha))^{\otimes} \rightarrow{ }^{\otimes} \Omega_{K}(\mathscr{R}(\beta))^{\otimes}$. We now have

If $S=\operatorname{colim}_{A} \mathscr{R}(\alpha)$, then $\Omega_{K}(S)=\operatorname{colim}_{A} \otimes \Omega_{K}(\mathscr{R}(\alpha))^{\otimes}$ (where the first colimit is in the category of $K$-rings and the second in the category of $S$-bimodules).

Construction 2.5. Tensor rings on $K$-bimodules. Let $B$ be a $K$-bimodule, and $S$ the tensor ring over $K$ :

$$
S=K\langle B\rangle=K \oplus B \oplus\left(B \otimes_{K} B\right) \oplus \cdots \text {. }
$$

Thus $S$ is generated as a $K$-ring by elements [b] corresponding to the elements $b \in B$, with the defining relations

$$
[a+b]=[a]+[b], \quad[\alpha b]=\alpha[b], \quad[b \alpha]=[b] \alpha \quad(a, b \in B ; \alpha \in K) .
$$

In view of (15) and (16) we may henceforth identify $B$ with its image in $K\langle B\rangle$, and drop the brackets around elements. From (16) it follows that $\Omega_{K}(S)$ will be generated as an $S$-bimodule by $\{d b \mid b \in B\}$ with relations

$$
d(a+b)=d a+d b, \quad d(\alpha b)=\alpha(d b), \quad d(b \alpha)=(d b) \alpha .
$$

This yields

For any $K$-bimodule $B$, one has $\Omega_{K}(K\langle B\rangle)={ }^{\otimes} B^{\otimes}$.

Now let $R$ be any $K$-ring. Then the coproduct of $K$-rings, $R \Perp K\langle B\rangle$, can be described as the tensor ring over $R$ on the $R$-bimodule $R \otimes B \otimes R$. Applying (13) to this coproduct we see that $\Omega_{K}(R\langle R \otimes B \otimes R\rangle)={ }^{\otimes} \Omega_{K}(R)^{\otimes} \oplus{ }^{\otimes} B^{\otimes}$. Note that here the tensor signs in the first summand refer to extension of scalars from $R$ to $S$, while those in the second summand refer to extension of scalars from $K$ to $S$. Let us put this in a form where both extensions are from $R$ to $S$ :

$$
\begin{aligned}
& \text { If } B \text { is a } K \text {-bimodule and } B^{\prime}=R \otimes B \otimes R \text {, then } \\
& \Omega_{K}\left(R\left\langle B^{\prime}\right\rangle\right)={ }^{\otimes} \Omega_{K}(R)^{\otimes} \oplus^{\otimes} B^{\prime \otimes} \text {. }
\end{aligned}
$$

Construction 2.6. Tensor rings on good R-bimodules. Formula 
(18) is in a way deceptive, for the map $d$ on $S=R\left\langle B^{\prime}\right\rangle$ does not carry the copy of $B^{\prime}$ in $S$ to the $B^{\prime}$ of the right-hand side, which represents $R(d B) R$. Rather, we see that an element of the form $a x b \in B^{\prime}(x \in B, a, b \in R)$ goes to $(d a) x b+a(d x) b+a x(d b)$, of which only the middle term belongs to this summand. Were it true that $d$ carried the $B^{\prime}$ on the left by the natural isomorphism to the $B^{\prime}$ on the right, then we would be able to use the fact that any $R$ bimodule is a of quotient a $K$-induced one to extend (18) to the case of arbitrary $R$-bimodules $B^{\prime}$; but the extended statement is easily shown to be false.

However, let us see how much is true. If $B$ is an $R$-bimodule, consider the natural map

$$
\Omega_{K}(R\langle B\rangle) \longrightarrow \Omega_{R}(R\langle B\rangle) .
$$

Comparing the definitions of the two terms, we see that the second $R\langle B\rangle$-bimodule will be obtainable from the first by imposing the relations $d r=0$ for all $r \in R$. Hence the map (19) is the cokernel of the map ${ }^{\otimes} \Omega_{K}(R)^{\otimes} \rightarrow \Omega_{K}(R\langle B\rangle)$. Also, by (17) the second bimodule of (19) is ${ }^{\otimes} B^{\otimes}$. So we have an exact sequence of $R\langle B\rangle$-modules

$$
{ }^{\otimes} \Omega_{K}(R)^{\otimes} \longrightarrow \Omega_{K}(R\langle B\rangle) \longrightarrow{ }^{\otimes} B^{\otimes} \longrightarrow 0 \text {. }
$$

Now let $\mathscr{B}$ denote the class of $R$-bimodules $B$ such that when " $0 \rightarrow$ " is added on the left the above sequence remains exact, and splits. This condition means that $\Omega_{K}(R\langle B\rangle)$ can be identified with $\otimes^{\otimes} \Omega_{K}(R)^{\otimes} \oplus{ }^{\otimes} B^{\otimes}$, in such a way that for $r \in R$, one has $d r=(d r, 0)$ while for $b \in B$, one has $d b=(*, d b)$. This is just what (18) tells us is true of any $R$-bimodule $B^{\prime}$ induced by a $K$-bimodule $B$, so such bimodules lie in $\mathscr{B}$.

It is easy to show from (14) that $\mathscr{B}$ is closed under arbitrary direct sums. For our applications we will also want the converse of this statement, namely

$$
\text { If } B_{1} \oplus B_{2} \in \mathscr{B} \text {, then } B_{1} \in \mathscr{B} \text {. }
$$

To get this, let $S=R\left\langle B_{1}\right\rangle, T=R\left\langle B_{1} \oplus B_{2}\right\rangle$. Since the symbol ${ }^{\otimes}()^{\otimes}$ could be ambiguous here, let us instead write ${ }^{S}()^{S}$ or ${ }^{T}()^{T}$, in each case referring to extension of scalars from $R$. We have obvious ring homomorphisms

$$
S \longrightarrow T \longrightarrow S
$$

composing to the identity. Using these we may identify $S$ with a subring of $T$, or regard $S$ as a $T$-ring.

Now applying $\Omega_{K}$ to (21), and the hypothesis $B_{1} \oplus B_{2} \in \mathscr{B}$, we get maps of $S$ - and $T$-bimodules, composing to the identity: 


$$
\Omega_{K}(S) \longrightarrow{ }^{T} \Omega_{K}(R)^{T} \oplus{ }^{T} B_{1}^{T} \oplus{ }^{T} B_{2}^{T} \longrightarrow \Omega_{K}(S) .
$$

The second map, going from a $T$-bimodule to an $S$-bimodule, factors through the result of changing scalars by $S \otimes_{T}() \otimes_{T} S$, so we get maps of $S$-bimodules composing to the identity

$$
\Omega_{K}(S) \longrightarrow{ }^{s} \Omega_{K}(R)^{s} \oplus{ }^{s} B_{1}^{S} \oplus{ }^{s} B_{2}^{S} \longrightarrow \Omega_{K}(S) .
$$

So $\Omega_{K}(S)$ will be isomorphic to the image of the left-hand arrow. Since $S$ is generated as a $K$-ring by $R$ and $B_{1}$, this image is the $S$-sub-bimodule of the middle term generated by $d(R)$ and $d\left(B_{1}\right)$. Recalling how $d$ behaves on $T$ (definition of $\mathscr{B}$ ) we can see that this image is precisely ${ }^{S} \Omega_{K}(R)^{S} \oplus{ }^{s} B_{1}^{S}$, with $d r=(d r, 0), d b=\left({ }^{*}, b\right)$. This proves $(20)$, and in fact we see

(23) In the context of (20), if $S=R\left\langle B_{1}\right\rangle$, then the behavior of the universal $K$-derivation $d: S \rightarrow \Omega_{K}(S)$ on the elements of $B_{1}$ may be computed by taking the universal derivation for $R\left\langle B_{1} \oplus B_{2}\right\rangle$, restricting to $B_{1}$, and replacing the $R\left\langle B_{1} \oplus B_{2}\right\rangle-$ coefficients in the resulting bimodule-expression by their images in $R\left\langle B_{1}\right\rangle$ (i.e., equating terms from $B_{2}$ to zero).

We shall see an example of how this works in the next subsection.

Construction 2.7. Adjoining morphisms of projective modules. We are now prepared to study the construction of freely adjoining to a ring $R$ a morphism $u: P^{\otimes} \rightarrow Q^{\otimes}$, where $P$ and $Q$ are two given finitely generated projective right $R$-modules. This will be the first step in several subsequent constructions.

As the discussion of $\S 2.1$ indicated, this is a generalization of the construction of adjoining an indeterminate, and can be achieved by adjoining a matrix $U$ of indeterminates and imposing certain matrix relations. Now when one adjoins indeterminates to a ring $R$ which is an algebra over a commutative ring $k$, one frequently wishes to make the indeterminates commute with $k$. So we should set up our results to allow us to impose such commutativity relations. In fact, the assumption that $R$ is a $k$-algebra can be weakened to certain commutativity conditions involving the presentations of the projective modules $P$ and $Q$.

Let $k$ be a ring (no connection yet with the $K$ of our earlier constructions) and $R$ a $k$-ring. By a $k$-structured projective right $R$-module we shall mean a $(k, R)$-bimodule $P$ which is embeddable as a bimodule direct summand in a direct sum of copies of the $(k, R)$-bimodule $R$. Note that a morphism ${ }^{m} R \rightarrow{ }^{n} R$ is a $(k, R)$ bimodule homomorphism if and only if the entries of the associated 
$n \times m$ matrix commute with $k$. Hence $k$-structured finitely generated projective $R$-modules can be represented as ranges of idempotent matrices $E$ over the centralizer of $k$ in $R$, and morphisms $a: E^{m} R \rightarrow$ $F^{n} R$ among such modules are represented by matrices $A$ over this centralizer, satisfying

$$
A E=A=F A \text {. }
$$

If we take $k=Z$, or more generally if $R$ is a $k$-algebra, then every projective right $R$-module has a unique $k$-structure, and every module homomorphism respects this structure. Hence the conventional concepts of projective modules are included in the concepts we are introducing. To signal that a morphism is to respect $k$-structure, we shall use an arrow with a subscript $k: a: P \rightarrow_{k} Q$.

Now, to adjoin to $R$ a k-centralizing indeterminate $x$ is equivalent to forming the tensor ring $R\langle B\rangle$ where $B$ is the $R$-bimodule freely generated by one $k$-centralizing generator $x$. This bimodule can be represented as $R \otimes_{k} R$, with the generator $1 \otimes 1$ called $x$. (Note that as $k$ is not assumed central in $R$, not all elements of this bimodule need be $k$-centralizing.) More generally, the result of adjoining to $R$ an $n \times m$ matrix $X$ of $k$-centralizing indeterminates $x_{i j}$ may be written $R\left\langle R^{n} \otimes_{k}{ }^{m} R\right\rangle$.

Let $P$ and $Q$ be finitely generated $k$-structured projective right $R$-modules, given by matrices $E, F$ as in (1). A morphism $a: P^{\otimes} \rightarrow Q^{\otimes}$ corresponds to a matrix $A$ of $k$-centralizing elements satisfying (2). To understand what happens when we impose (2) on a matrix of indeterminates, we need some general observations:

If $X=\left(\left(x_{i j}\right)\right)$ is an $n \times m$ matrix of elements of any $R$-bimodule $B$, (for example, of an $R$-ring) we have the decomposition

$$
X={ }_{F} X_{E}+{ }_{F} X_{E^{\prime}}+{ }_{F^{\prime}} X_{E}+{ }_{F^{\prime}} X_{E^{\prime}},
$$

where we define

$$
\begin{array}{rlrl}
{ }_{F} X_{E} & =F X E, & { }_{F} X_{E^{\prime}} & =F X(I-E), \\
{ }_{F^{\prime}} X_{E} & =(I-F) X E, \quad{ }_{F^{\prime}} X_{E^{\prime}}=(I-F) X(I-E) .
\end{array}
$$

Here we note

$$
\begin{array}{rlrl}
{ }_{F} X_{E} & =F_{F} X_{E} E, & { }_{F} X_{E^{\prime}} & =F_{F} X_{E^{\prime}}(I-E), \\
{ }_{F^{\prime}} X_{E} & =(I-F)_{F^{\prime}} X_{E} E,{ }_{F^{\prime}} X_{E^{\prime}}=(I-F)_{F^{\prime}} X_{E^{\prime}}(I-E) .
\end{array}
$$

Conversely, if we are given any four $n \times m$ matrices ${ }_{E} X_{F},{ }_{F}, X_{E}$, ${ }_{F} X_{E^{\prime}},{ }_{F}^{\prime} X_{E^{\prime}}$ satisfying (26), and we define $X$ by (24), we see that (25) will hold. Thus, to give four matrices over $B$ satisfying (26) is equivalent to giving one arbitrary matrix, via the correspondence established by (24) and (25). Further, $X$ will be $k$-centralizing if 
and only if ${ }_{F} X_{E},{ }_{F}, X_{E},{ }_{F} X_{E^{\prime}}$, and ${ }_{E^{\prime}} X_{F^{\prime}}$ all are, since $E$ and $F$, the matrices defining $P$ and $Q$, were assumed $k$-centralizing.

It follows that if we let ${ }_{F} C_{E}$ denote the $R$-bimodule generated by a universal $n \times m$-tuple of $k$-centralizing elements forming a matrix ${ }_{F} X_{E}$ satisfying ${ }_{F} X_{E}=F_{F} X_{E} E$, and if ${ }_{F} C_{E^{\prime}},{ }_{F}, C_{E},{ }_{F}, C_{E^{\prime}}$ are defined analogously, then the $R$-bimodule with a universal unrestricted $n \times m$-tuple of $k$-centralizing elements $X$ will be their direct sum, under the correspondence given by (24) and (25). Thus, ${ }_{F} C_{E} \oplus_{F} C_{E^{\prime}}$ $\bigoplus_{F^{\prime}} C_{E} \bigoplus_{F^{\prime}} C_{E^{\prime}} \cong R^{n} \otimes_{k}{ }^{m} R$.

Let $Q^{*}$ denote the $k$-structured projective left $R$-module $R^{n} F$ (that is, the image of $R^{n}$ under the right action of $F$, isomorphic to $\operatorname{Hom}_{R}(Q, R)$ made an $(R, k)$-bimodule in the natural way). Then one easily verifies

The $R$-bimodule (called ${ }_{F} C_{E}$ above) presented by an $n \times m$-tuple of $k$-centralizing generators $u_{i j}$ subject to the matrix relation $U=F U E$ (equivalently $F U=U=U E$ ) embeds as a direct summand in the $R$-bimodule freely generated by an $n \times m$ tuple of $k$-centralizing generators $x_{i j}$, via the identification $U=F X E$. Its image can be written

$$
\left(R^{n} F\right) \otimes_{k}\left(E^{m} R\right)=Q^{*} \otimes_{k} P \subseteq\left(R^{n}\right) \otimes_{k}\left({ }^{m} R\right) .
$$

Hence,

The ring obtained by freely adjoining to $R$ a morphism of $k$-structured projective right modules, $S=R\left\langle u: P^{\otimes} \rightarrow_{k} Q^{\otimes}\right\rangle$ can be written $R\left\langle Q^{*} \otimes_{k} P\right\rangle$. In fact, the isomorphism $R\left\langle Q^{*} \otimes_{k} P\right\rangle \widetilde{\rightarrow} R\left\langle u: P^{\otimes} \rightarrow_{k} Q^{\otimes}\right\rangle$ is given by $q \otimes p \mapsto q u p(p \in$ $P \subseteq P^{\otimes}, q \in Q^{*} \subseteq\left(Q^{\otimes}\right)^{*}$.)

(Digression: It may appear that there is something wrong with the variances in (27) and (28). To see that they are correct, note that the tensor algebra $S=R\left\langle Q^{*} \otimes_{k} P\right\rangle$ is characterized by having a universal $R$-bimodule map $v: Q^{*} \otimes_{k} P \rightarrow S$. This clearly has the correct variances in $P$ and $Q$ to correspond to a map $u: P^{\otimes} \rightarrow_{k} Q^{\otimes}$. What is causing the confusion is the notorious duality between elements and coordinates! As a vague but useful principle, when one performs a universal construction, the object constructed is covariant in the system of elements adjoined, but contravariant in the item for which these are to be universal coordinates. The trouble is that our notation sometimes shows this item ( $u$ above) and other times does not ( $v$ above). The reader may wish to check the following simpler case: If $R$ is a commutative ring and $P$ a finitely generated projective $R$-module, then the commutative $R$-algebra with a universal element of $P^{\otimes}$, which we could call 
$R\left[x \in P^{\otimes}\right]$, is the symmetric algebra $\left.R[P *].\right)$

Now once again suppose that $R$ is a $K$-ring as in previous constructions; and take for the $k$ of the above discussion a subring of $K$. Then the $R$-bimodule $\left(R^{n} \otimes_{k}{ }^{n} R\right)$ is induced by extension of scalars from the $K$-bimodule $\left(K^{n} \otimes_{k}{ }^{m} K\right)$. Thus first (18) gives

If $S=R\langle X\rangle$ is the $R$-ring universally generated by an $n m$-tuple of $k$-centralizing elements $x_{i j}$, then $\Omega_{K}(S) \cong$ ${ }^{\otimes} \Omega_{K}(R)^{\otimes} \oplus\left(S^{n} \otimes_{k}{ }^{m} S\right)$.

And applying (28) and (20) to the above we get

If $P$ and $Q$ are $k$-structured finitely generated projective right $R$-modules, and $S=R\left\langle u: P^{\otimes} \rightarrow_{k} Q^{\otimes}\right\rangle$ is the extension of $R$ by a universal $k$-structured module morphism between them, then $\Omega_{K}(S) \cong{ }^{\otimes} \Omega_{K}(R)^{\otimes} \oplus^{\otimes} Q * \otimes_{k} P^{\otimes}$. The summand ${ }^{\otimes} Q * \otimes_{k} P^{\otimes}$ is generated by the entries of the matrix $Z=F(d U) E$, which are $k$-centralizing and subject to the relation $Z=F Z E$. The action of $d$ on $S=R\langle u\rangle$ extends the action on $R$, and is described on the entries of $U$ by

$$
d U=(d F) U+Z+U(d E) .
$$

To get (31) we follow the prescription in (23), and in $R\langle X\rangle$ compute $d(F X E)=(d F) X E+F(d X) E+F X(d E)$, then substitute $U$ for $X$ and $Z$ for $F(d U) E$.

Given $E$ and $F$ as above, we shall call a matrix $U$ of elements of a $R$-bimodule an $(F, E)$-matrix if it satisfies $U=F U E$, an $\left(F^{\prime}, E\right)$-matrix if $(I-F) U E=U$, etc.; and call ${ }_{F} X_{E}$ the $(F, E)$ component of $X$, etc. It can be suggestive to think of these components into which we decompose a matrix as "blocks". For example a matrix whose decomposition (24) is $A+B+C+D$ might be thought of as $\left(\begin{array}{ll}A & B \\ C & D\end{array}\right)$. (But we shall not make important use of this idea because in the absence of additional conventions it conflicts with usual matrix notation.)

Note that if we combine (30) with (4) we get:

If $P$ and $Q$ are $k$-structured finitely generated projective right $R$-modules, and $S=R\left\langle u, u^{-1}: P^{\otimes} \cong Q^{\otimes}\right\rangle$ is the ring obtained by universally adjoining to $R$ a $k$-structured module isomorphism between them, then $\Omega_{K}(S) \cong{ }^{\otimes} \Omega_{K}(R)^{\otimes} \oplus{ }^{\otimes} P * \otimes_{k} P^{\otimes}$.

Construction 2.8. Adjoining idempotent module-endomorphisms. Let $k$ be any subring of $K$, and $m$ a positive integer. Suppose we adjoin to our $K$-ring $R$ an $m \times m$ matrix of $k$-centralizing indeterminates, $E$, and further impose the relation 


$$
E^{2}=E \text {. }
$$

Thus, over the resulting ring $S$, we have a universal decomposition of ${ }^{m} S$ into a direct sum of two $k$-structured projective modules,

$$
{ }^{m} S=P \oplus Q, \text { where } P=E^{m} S, \quad Q=(I-E)^{m} S .
$$

We see that $\Omega_{K}(S)$ will be obtained by adjoining to ${ }^{\otimes} \Omega_{K}(R)^{\otimes}$ an $m \times m$ matrix $d E$ of $k$-centralizing bimodule-generators, and the relations

$$
(d E) E+E(d E)=d E .
$$

To study (34), let us take its $(E, E)-,\left(E, E^{\prime}\right)-,\left(E^{\prime}, E\right)$ - and $\left(E^{\prime}, E^{\prime}\right)$-components. Only the first and last of these turn out to be nonvacuous; these are respectively

$$
E(d E) E=0, \quad(I-E)(d E)(I-E)=0 .
$$

This says that $d E$ has "block" form $\left(\begin{array}{ll}0 & * \\ * & 0\end{array}\right)$ in the language of the end of the last subsection. We should pause to note that the above computation holds for any idempotent matrix $E$ over any ring. For instance, we may apply it to both $E$ and $F$ in (31). Recalling that $U$ was an $(F, E)$-matrix, we conclude that the term $(d F) U$ of (31) represents an $\left(F^{\prime}, E\right)$-matrix over $\Omega_{K}(S)$, and $U(d E)$ an $\left(F, E^{\prime}\right)$ matrix, so (31) has the "block" form:

$$
d U=\left(\begin{array}{lc}
Z & U(d E) \\
(d F) U & 0
\end{array}\right)
$$

Returning to the universal case of (35), we see that as we have no further equations, the two other components $E(d E)(I-E)$ and $(I-E)(d E) E$ will be universal $k$-centralizing $\left(E, E^{\prime}\right)$ - and $\left(E^{\prime}, E\right)$ matrices of $S$-bimodule elements. Calling these $Z$ and $Z^{\prime}$, we get

If $S$ is the $R$-ring obtained by adjoining to $R$ a universal $k$-centralizing idempotent $m \times m$ matrix $E$, and we define $P=E^{m} S, Q=(I-E)^{m} S$, then

$$
\Omega_{K}(S)={ }^{\otimes} \Omega_{K}(R)^{\otimes} \oplus\left(P^{*} \otimes_{k} Q\right) \oplus\left(Q^{*} \otimes_{k} P\right) .
$$

If we write $Z$ and $Z^{\prime}$ for the canonical $\left(E, E^{\prime}\right)$ - and $\left(E^{\prime}, E\right)$ matrices of generators of the last two bimodule summands, then the universal derivation $d$ is determined by $d E=Z+Z^{\prime}$.

As a more general case, we may adjoin to $R$ an idempotent endomorphism $E$ of an already existing $k$-structured finitely generated projective right $R$-module $P=F^{m} R$, getting a universal splitting $P=P_{1} \oplus P_{2}=E^{m} R \oplus(F-E)^{m} R$. Here the defining relations are 


$$
\begin{aligned}
& E=F E F \\
& E=E^{2}
\end{aligned}
$$

Now by (30) and (31), equation (38) alone yields a decomposition $d E=(d F) E+Z+E(d F)$ where $Z$ is an $(F, F)$-matrix. On the other hand, (39) yields (35), into which we substitute the above formula. By reasoning essentially like that which gave (37) we get

(40) Let $P=F^{m} R$ be a $k$-structured projective right $R$-module, and $S$ the $R$-ring with a universal decomposition $P^{\otimes}=$ $P_{1} \oplus P_{2}=E P^{\otimes} \oplus(F-E) P^{\otimes}$. That is, $S=R\left\langle e: P^{\otimes} \rightarrow_{k} P^{\otimes} \mid e^{2}=e\right\rangle$. Then $\Omega_{K}(S)={ }^{\otimes} \Omega_{K}(R)^{\otimes} \oplus\left(P_{1}^{*} \otimes_{k} P_{2}\right) \oplus\left(P_{2}^{*} \otimes_{k} P_{1}\right)$. If we write $Z_{1}$ and $Z_{2}$ for the canonical $(E, F-E)$ - and $(F-E, E)$ matrices of generators of the last two bimodule summands, then $d E=(d F) E+Z_{1}+Z_{2}+E(d F)$. Here the block form, in terms of the decomposition $I_{m}=E+(F-E)+(I-F)$, is

$$
d E=\left(\begin{array}{ccc}
0 & Z_{1} & (d F) E \\
Z_{2} & 0 & 0 \\
E(d F) & 0 & 0
\end{array}\right)
$$

Construction 2.9. One-sided localization. Again let $P=E^{m} R$, $Q=F^{n} R$ be $k$-structured finitely generated projective right $R$-modules, and let us be given a morphism $a: P \rightarrow_{k} Q$, described by an $(F, E)$ matrix $A$. Suppose we adjoin to $R$ a universal left inverse $u$ of $a$, to get the $R$-ring $S=R\left\langle u: Q^{\otimes} \rightarrow_{k} P^{\otimes} \mid u a=1_{P} \otimes\right\rangle$. This means adjoining an $m \times n$ matrix of $k$-centralizing indeterminates, $U$, and imposing the relations

$$
\begin{gathered}
U=E U F, \\
U A=E .
\end{gathered}
$$

The corresponding equations in $\Omega_{K}(S)$ are

$$
\begin{aligned}
d U= & (d E) U+E(d U) F+U(d F), \\
& (d U) A+U(d A)=d E .
\end{aligned}
$$

Now we have seen that the adjunction of $U$ satisfying (41) alone enlarges $\Omega_{K}(R)$ by a direct summand ${ }^{\otimes} P^{*} \otimes_{k} Q^{\otimes}$, whose $(E, F)$ matrix of generators is the middle term of (43). If we now substitute (43) into (44), and make use of the properties of $d E, d A$, etc., as well as the hypotheses on $U, A$ etc., we find that the resulting equation is equivalent to

$$
E(d U)(A U)=-U(d A) U \text {. }
$$

Now notice that because $u$ is a left inverse to $a$, the product 
$a u$ is an idempotent endomorphism of $Q^{\otimes}=F^{n} S$, giving a splitting $Q^{\otimes}=Q_{1} \oplus Q_{2}=(A U)^{n} S \oplus(F-A U)^{n} S$. We now see that (45) takes the originally universal $(E, F)$-matrix $E(d U) F$, and equates its $(E, A U)$-component with an $(E, A U)$-matrix having entries in ${ }^{\otimes} \Omega_{K}(R)^{\otimes}$. This "removes" part of our summand ${ }^{\otimes} P^{*} \otimes_{k} Q^{\otimes}$, leaving only ${ }^{\otimes} P^{*} \otimes_{k} Q_{2}$. In summary:

(46) Let $P=E^{m} R$ and $Q=F^{n} R$ be finitely generated $k$-structured projective right $R$-modules, and $a: P \rightarrow Q$ a $(k, R)$-morphism. Over $S=R\left\langle u: Q^{\otimes} \rightarrow_{k} P^{\otimes} \mid u a=1_{P} \otimes\right\rangle$, let $Q^{\otimes}=Q_{1} \oplus Q_{2}=$ $(a u) Q^{\otimes} \oplus(1-a u) Q^{\otimes}$. Then $\Omega_{K}(S)={ }^{\otimes} \Omega_{K}(R)^{\otimes} \oplus{ }^{\otimes} P^{*} \otimes_{k} Q_{2}$. Here the second summand is generated by the entries of the $(E, F-A U)$-matrix $Z=E(d U)(F-A U)$. Further

$$
d U=(d E) U-U(d A) U+Z+U(d F) .
$$

REMARK. Suppose a already has a right inverse $v$ over $R$. Then adjoining a left inverse is equivalent to adjoining a 2 -sided inverse. Then we will get $a u=1_{Q} \otimes$, so $Q_{2}$ and $F-A U$ are zero, so (46) and (47) reduce to (4) and (9). In particular, the description of $\Omega_{K}(S)$ ceases to depend on $k$, which is why there was no loss of generality in treating 2 -sided inversion before introducing $k$-structure. (The general principle is that if a morphism $a$ in any category satisfies an equation $\alpha a=a \alpha^{\prime}$, then a 2-sided inverse to $a$ will satisfy $\left(a^{-1}\right) \alpha=\alpha^{\prime}\left(a^{-1}\right)$. Hence if a module-morphism $a$ respects $k$-structure on $P$ and $Q$ for some $k$, any 2 -sided inverse to $a$ will automatically do the same, so it makes no difference to specially impose such conditions.)

2.10. Coproducts, matrices, antimatrices, and products. We shall end this section of the paper with a few results that will not be used in our main result, but which are not hard to derive. We will be less detailed in our arguments than above. We begin with some constructions that change not only " $R$ " but also " $K$ ".

First a generalization of (14). Let $A$ be a small category, and let us be given two functors $\mathscr{C}$ and $\mathscr{R}$ from $A$ into the category of rings, and a morphism $\mathscr{K} \rightarrow \mathscr{R}$. Thus, for each $\alpha, \mathscr{R}(\alpha)$ is a $\mathscr{\mathscr { C }}(\alpha)$-ring. We easily see

$$
\begin{aligned}
& \text { If we write } L=\operatorname{colim}_{A} \mathscr{\mathscr { L }}(\alpha), \quad S=\operatorname{colim}_{A} \mathscr{R}(\alpha), \quad \text { then } \\
& \Omega_{L}(S)=\operatorname{colim}_{A} \otimes \Omega_{\mathscr{K}^{\prime}(\alpha)}(\mathscr{R}(\alpha))^{\otimes} \text {. }
\end{aligned}
$$

Next, let $K$ be a ring, and $K^{\prime}, R$ two $K$-rings.

$$
\text { If we put } S=R \Perp_{K} K^{\prime} \text {, then } \Omega_{K^{\prime}}(S)=\otimes^{\otimes} \Omega_{K}(R)^{\otimes} \text {. }
$$


This may either be seen directly by "generators and relations", or gotten from (48) by taking for $A$ the diagram $\bullet \leftarrow \cdot \rightarrow \cdot$, for $\mathscr{K}$ the functor $K \leftarrow K \rightarrow K^{\prime}$, and for $\mathscr{R}$ the functor $R \leftarrow K \rightarrow K^{\prime}$.

The behavior of universal derivations on a matrix ring $M_{n}(R)$ is likewise easily described if we simultaneously apply $M_{n}$ to the base ring $K$. Considering $M_{n}(R)$ an $R$-ring by the diagonal map, we have

$$
\begin{aligned}
& \Omega_{M_{n}(K)}\left(M_{n}(R)\right)={ }^{\otimes} \Omega_{K}(R)^{\otimes}=M_{n}\left(\Omega_{K}(R)\right) \text {, with } d: M_{n}(R) \rightarrow M_{n}\left(\Omega_{K}(R)\right) \\
& \text { given by entry-wise application of } d: R \rightarrow \Omega_{K}(R) .
\end{aligned}
$$

Indeed, the homomorphism $\left(1_{R}, d\right): R \rightarrow\left(R, \Omega_{K}(R)\right)$ induces a homomorphism $M_{n}(R) \rightarrow M_{n}\left(\left(R, \Omega_{K}(R)\right)\right)=\left(M_{n}(R), M_{n}\left(\Omega_{K}(R)\right)\right)$, which is easily shown to have the correct universal property.

Now let $R$ be a $K$-ring and $n$ a positive integer, and let $S$ denote the coproduct, over $K$, of $R$ with the matrix ring $M_{n}(K)$. Then $S$ is an $M_{n}(K)$-ring. But any $M_{n}(K)$-ring has the form of $n \times n$ matrices over a $K$-ring. So we can write $S=M_{n}(T)$ for some $K$-ring $T$. Following [3], $\S 7, T$ will be called $\mathfrak{w}_{n}(R)$. The functor $\mathfrak{w}_{n}$ is a left adjoint to the functor $M_{n}$ from $K$-rings to $K$-rings. (The "antimatrices" of the title of this subsection.) That is, $\mathfrak{w}_{n}(R)$ is universal among all $K$-rings $T^{\prime}$ with a $K$-ring homomorphism $R \rightarrow M_{n}\left(T^{\prime}\right)$. Equivalently, $T$ is such that there is a universal representation of the $K$-ring $R$ by endomorphisms of the right $T$-module ${ }^{n} T$, or of the left $T$-module $T^{n}$.

Since $M_{n}(T)=S=R \Perp M_{n}(K)$, we can apply both (49) and (50). Together they give an $S$-bimodule isomorphism

$$
M_{n}\left(\Omega_{K}(T)\right) \cong{ }^{\otimes} \Omega_{K}(R)^{\otimes} .
$$

To get from (51) a description of $\Omega_{K}(T)$ we need to know how, for any ring $T$, one recovers from an $M_{n}(T)$-bimodule $M_{n}(B)$ the $T$-bimodule $B$. In fact, this is achieved by tensoring on the left by the $\left(T, M_{n}(T)\right)$-bimodule $T^{n}$ and on the right by the $\left(M_{n}(T), T\right)$ bimodule ${ }^{n} T$. When we apply this to the right side of (51), which arises by extending scalars from $R$ to $M_{n}(T)=S$, the effect is that the above $T^{n}$ and ${ }^{n} T$ are considered as $(T, R)$ - and $(R, T)$-bimodules, via the universal representation of $R$ over $T$ referred to above. So we get:

(52) Let $T=\mathfrak{w}_{n}(R)$. Denote the universal representation $R \rightarrow$ $M_{n}(T)$ by $r \mapsto\left(\left(r_{i j}\right)\right)$ (so that for $r \in R, r_{11}, \cdots, r_{n n} \in T$ ) and the canonical right and left $T$-bases of ${ }^{n} T$ and $T^{n}$ by $\varepsilon_{i}, \eta_{j}$. Then $\Omega_{K}(T)=T^{n} \otimes_{R} \Omega_{K}(R) \otimes_{R}{ }^{n} T$, and the universal $K$-derivation $d$ on $T$ is characterized by 


$$
d\left(r_{i j}\right)=\varepsilon_{i} \otimes\left(d_{R} r\right) \otimes \eta_{j} .
$$

The behavior of $\Omega_{K}$ on finite direct products, as on matrix rings, is very simple if we simultaneously apply the construction to the base rings $K$. We claim

If $R_{1}$ is a $K_{1}$-ring and $R_{2}$ is a $K_{2}$-ring, and we consider $R_{1} \times R_{2}$ as a $K_{1} \times K_{2}$-ring, then $\Omega_{K_{1} \times K_{2}}\left(R_{1} \times R_{2}\right)=\Omega_{K_{1}}\left(R_{1}\right) \oplus \Omega_{K_{2}}\left(R_{2}\right)$.

Note that this statement that our $\Omega_{K_{1} \times K_{2}}$ is the direct sum of an $\left(R_{1}, R_{1}\right)$-bimodule and an $\left(R_{2}, R_{2}\right)$-bimodule means that, if we write $E=(1,0) \in R_{1} \times R_{2}$, then our bimodule is spanned by just its $(E, E)$ elements and $\left(E^{\prime}, E^{\prime}\right)$-elements (degenerate case of the term " $(F, E)$ matrix"). This follows from the corresponding fact for the ring $R_{1} \times R_{2}$ itself, together with the fact that $E$ and $1-E$ lie in $K_{1} \times K_{2}$, so that $d E$ and $d(1-E)$ are zero. (See (31), which holds for any $(F, E)$-matrix $U$.$) On the other hand, when we take a direct$ product of $K$-rings over a fixed $K$, we also get $\left(E, E^{\prime}\right)$ and $\left(E^{\prime}, E\right)$ parts. The reader can verify

If $R$ and $S$ are $K$-rings, then $\Omega_{K}(R \times S)=\Omega_{K}(R) \oplus\left(S \otimes_{K} R\right) \oplus$ $\left(R \otimes_{K} S\right) \oplus \Omega_{K}(S)$. Writing this direct sum as a matrix $\left(\begin{array}{cc}\Omega(R) & R \otimes S \\ S \otimes R & \Omega(S)\end{array}\right)$ we can describe the universal derivation on $R \times S$ by:

$$
d(r, 0)=\left(\begin{array}{cc}
d r & r \otimes 1 \\
-1 \otimes r & 0
\end{array}\right), \quad d(0, s)=\left(\begin{array}{cc}
0 & -1 \otimes s \\
s \otimes 1 & d s
\end{array}\right) .
$$

More generally, if $S=R_{1} \times \cdots \times R_{n}$, then $\Omega_{K}(S)$ is a direct sum of $n^{2}$ bimodules, namely the $\left(R_{i}, R_{i}\right)$-bimodules $\Omega_{K}\left(R_{i}\right)(i \leqq n)$ and the $\left(R_{i}, R_{j}\right)$-bimodules $R_{i} \otimes_{K} R_{j}(i \neq j)$.

Finally, let us obtain a description of $\Omega_{K}\left(M_{n}(R)\right)$, where $R$ is a $K$-ring and $n$ a positive integer. Note that we may construct $M_{n}(R)$ as follows. Start with the $K$-ring

$$
S=R \times K \times \cdots \times K \quad \text { (one } R \text { and } n-1 K^{\prime} \mathrm{s} \text { ). }
$$

Let us write $e_{i i} \in S$ for the idempotent with 1 in the $i$ th place and zeroes elsewhere, in anticipation of the way $S$ is to be mapped into our matrix ring. Now universally adjoin $n-1$ isomorphisms of $K$-structured projective right $S$-modules, $e_{1 i}: e_{i i} S^{\otimes} \rightarrow_{K} e_{11} S^{\otimes}(1<i \leqq n)$, with inverses which we shall denote $e_{i 1}: e_{11} S^{\otimes} \rightarrow_{K} e_{i i} S^{\otimes}$. The verification that the resulting $K$-ring is $M_{n}(R)$ is not difficult. (The element $r e_{i j}(r \in R)$ can be obtained as $\left.e_{i 1} \cdot e_{11} r \cdot e_{1 j} \cdot\right)$

Now by (57) and the preceding discussion, $\Omega_{K}(S)$ is the direct sum of $\Omega_{K}(R)$ and the $n^{2}-n$ bimodules $S e_{i i} \otimes_{K} e_{j j} S$. When we 
adjoin the $e_{i 1}$ 's and their inverses, (32) tells us that this has the effect of extending scalars and throwing in $n-1$ bimodules ${ }^{\otimes} e_{i i} \otimes_{K} e_{11} S^{\otimes}$. But our extension process has also made all the projective modules $e_{i i} S^{\otimes}$ isomorphic, and likewise for the corresponding left modules. Hence the $\left(n^{2}-n\right)+(n-1)$ summands that have been added in to our universal derivations bimodule become isomorphic:

(58) Let $R$ be a $K$-ring, $T=M_{n}(R)$, and let $P$ denote the projective $T$-module $e_{11} T$. Then $\Omega_{K}(T) \cong{ }^{\otimes} \Omega_{K}(R)^{\otimes} \oplus\left(P^{*} \bigotimes_{K} P\right)^{n^{2}-1} \cong$ $M_{n}\left(\Omega_{K}(R) \oplus\left(R \otimes_{K} R\right)^{n^{2}-1}\right)$.

2.11. REMARKS. The reader may have noticed that in the formulas by which we described the action of a universal derivation $d$ on the morphisms introduced in our universal constructions, there were generally just one or two terms involving the new generators of $\Omega_{K}(S)$, and a large number of "automatic" terms. An appendix to this paper, $\S 7$, describes a formalism for working with derivations on morphisms of projective modules, by which one can eliminate most of these "automatic" terms, and thus put those equations in simpler form.

Let us note that not every important $K$-ring construction $S$ yields a conveniently describable $\Omega_{K}(S)$. Examples of some constructions for which there does not seem to exist a nice description of this bimodule are:

(a) The tensor product, over a commutative ring $K$, of two given $K$-algebras.

(b) Infinite direct products $\Pi R_{i}$; formal power series rings $R \llbracket t]$. For these we doubt that there is even a reasonable normal form description of $\Omega_{K}(S)$.

(c) The construction $(R, B)=R\langle B\rangle /\left(B^{2}\right)$-ironical in view of the importance of this construction in characterizing and studying $\Omega_{K}(R)$ (cf. $\left.\S 1,2.1\right)$ !

(However, there is always a "nice" description of $\Omega_{K}(R)$ in another sense: that given by (61) below. In fact, we shall use the results of this section by playing them off against that description.)

Observe that $\Omega_{K}(R)$ will always be generated as a right $R$-module by its $K$-subbimodule $d R$ (which by definition generates it as an $R$-bimodule). This follows from the formula

$$
a(d b)=d(a b)-(d a) b .
$$

The following description of $\Omega_{K}(R)$, which is obtained from the splitting of (61) below, will not be used here but may be of interest: 
(60) Let $R$ be a $K$-ring. Then as $(K, R)$-bimodule, $\Omega_{K}(R) \cong$ $(R / K) \bigotimes_{K} R$, where $d r=(r+K) \otimes 1$. The left $R$-module structure of $\Omega_{K}(R)$ is determined by (59).

As a consequence, if $R \cong S$ is a $K$-bimodule direct summand, $S=R \oplus B$, then as $(K, S)$-bimodule, $\Omega_{K}(S) \cong \Omega_{K}(R)^{\otimes} \oplus\left(B \otimes_{K} S\right)$.

3. Applications: Hereditary rings, and Schreier formulas. Let $K \rightarrow R$ be a ring homomorphism. The usefulness of the universal derivation bimodule $\Omega_{K}(R)$ to us will come from the following exact sequence ([11], Proposition IX. 3.2; cf. [10], p. A III. 132 or [6]).

$$
0 \longrightarrow \Omega_{K}(R) \longrightarrow R \otimes_{K} R \longrightarrow R \longrightarrow 0 \text {. }
$$

Here the first map takes $d r$ to $r \otimes 1-1 \otimes r$, and the second is multiplication.

Since the last term of (61) is projective as a left $R$-module, (61) is left $R$-split, so for any right $R$-module $M$, (61) remains exact under $M \otimes_{R}$. This gives us a presentation of $M$ :

$$
0 \longrightarrow M \otimes_{R} \Omega_{K}(R) \longrightarrow M \otimes_{K} R \longrightarrow M \longrightarrow 0 \text {. }
$$

Suppose $M \otimes_{K} R$ is projective as right $R$-module. Then $M$ will have homological dimension $\leqq 1$ if and only if $M \otimes_{R} \Omega_{K}(R)$ is projective. Let us record the result in the case where $M \otimes_{K} R$ is projective for all $M$. We recall that a ring $L$ has all right modules projective if and only if $L$ is a semisimple Artin ring, that is, a finite direct product of matrix rings over skew fields.

Proposition 3.1. Let $R$ be a K-ring. Suppose that $K$ is semisimple Artinian (or more generally, that the map $K \rightarrow R$ factors through a semisimple Artin ring, or still more generally, that for every right $K$-module $H$, the right $R$-module $H \otimes_{K} R$ is projective).

Then a right $R$-module $M$ has homological dimension $\leqq 1$ if and only if $M \otimes_{R} \Omega_{K}(R)$ is projective.

In particular, $R$ is right hereditary if and only if $M \otimes_{R} \Omega_{K}(R)$ is projective for every right $R$-module $M$.

Note that under the hypotheses of the above Proposition, if $k$ is a subring of $K$, then for every right $k$-module $H$, we have $H \otimes_{k} R=\left(H \otimes_{k} K\right) \otimes_{K} R$ projective. It is easily deduced that if $P$ is any $k$-structured projective right $R$-module, $H \otimes_{k} P$ will likewise be projective.

We now set things up so that we can apply the results of the preceding section: 
COROLLARY 3.2. Let $K$ be a ring, $\left(R_{\alpha}\right)_{\alpha \in A}$ a nonempty family of $K$-rings each satisfying the condition of the first paragraph of Proposition 3.1, and $S$ a K-ring given with K-ring homomorphisms $\varphi_{\alpha}: R_{\alpha} \rightarrow S$. Suppose that $\Omega_{K}(S)$ can be written as a direct sum of copies of the S-bimodules ${ }^{\otimes} \Omega_{K}\left(R_{\alpha}\right)^{\otimes}$ and of S-bimodules of the form $N \otimes_{k} P$, where the $k$ are subrings of $K$, the $N$ are $(S, k)$-bimodules, and the $P$ are $k$-structured projective right $S$-modules.

Then $S$ again satisfies the conditions of Proposition 3.1, and if a right $S$-module $M$ has homological dimension $\leqq 1$ over each $R_{\alpha}$, it has homological dimension $\leqq 1$ over $S$.

In particular, if all $R_{\alpha}$ are right hereditary then so is $S$.

Proof. The first conclusion states that if $H$ is any right $K$-module then $H \otimes_{K} S$ is a projective $S$-module. And indeed, choosing any $\alpha \in A$ we can write $H \otimes_{K} S$ as $\left(H \otimes_{K} R_{\alpha}\right) \otimes_{R_{\alpha}} S$ and apply our hypothesis on $R_{\alpha}$ to $H \otimes_{K} R_{\alpha}$.

Now if $M$ is a right $S$-module, $M \otimes_{S} \Omega_{K}(S)$ will be a direct sum of modules of the forms $M \otimes_{S}\left({ }^{\otimes} \Omega_{K}\left(R_{\alpha}\right)^{\otimes}\right)$ and $M \otimes_{S}\left(N \otimes_{k} P\right)$. Those of the first sort can be rewritten $M \otimes_{R_{\alpha}} \Omega_{K}\left(R_{\alpha}\right)^{\otimes}$, and hence are projective if $M$ has $R_{\alpha}$-homological dimension $\leqq 1$. Those of the latter sort can be written $\left(M \otimes_{S} N\right) \bigotimes_{k} P$, and so are projective by our preceding observations.

REMARK. We have allowed possibly more than one $R_{\alpha}$ in order to cover the coproducts construction.

In stating the remaining consequences, we shall for brevity assume $K$ semisimple Artinian, though the more general hypothesis of Proposition 3.1 could be retained. We shall also refrain from restating results proved in [2] and [3].

First, our promised main result. Note that if an $R$-module is referred to as $k$-structured, morphisms are understood to mean $(k, R)$ homomorphisms.

THEOREM 3.3. Let $K$ be a semisimple Artin ring and $R$ a $K$-ring. Then if $R$ is hereditary, so is any ring $S$ obtained from $R$ by universal (2-sided) inversion of any family of morphisms of finitely generated projective $R$-modules, and universal right and left inversions of families of morphisms of k-structured projective $R$-modules, for subrings $k$ of $K$.

Next, we take advantage of the good behavior of $\Omega_{K}$ under colimits (14): 
THEOREM 3.4. Let $K$ be a semisimple Artin ring. Then any $K$-ring constructed from right hereditary $K$-rings $R_{\alpha}$ by a possibly transfinite sequence of the following steps is again right hereditary. (The $k$ of (i)-(iii) refers to any subring of $K$. Like $P$ and $Q$, it can vary from step to step. At steps indexed by limit ordinals one forms the direct limit.)

(i) Adjoining a universal map between k-structured projective modules: $u: P^{\otimes} \rightarrow_{k} Q^{\otimes}$.

(ii) Universally splitting a k-structured projective module: $P^{\otimes}=P_{1} \oplus P_{2}$.

(iii) Adjoining a universal left, right or 2-sided inverse to a given morphism of finitely generated $k$-structured projective modules.

(iv) Forming coproducts of $K$-rings.

In |3|, Theorems 6.2 and 6.4 , a generally infinite sequence of steps (ii), (i) and (iii) was used to obtain rings $S$ whose semigroup of isomorphism classes of finitely generated projective modules, with distinguished element [S], was an arbitrary abelian semigroup with distinguished element, satisfying two obvious necessary conditions. It followed from the results of that paper that if the given semigroup was finitely generated, $S$ would be hereditary. The above result shows that $S$ will be hereditary in all cases." Thus in [3] one may drop the words "finitely generated" from Theorem 6.2, and delete Theorem 6.4 and Question 6.5.

REMARK 3.5. Variant results. It is easy to see from the proof that Proposition 3.1 will remain valid if, in the last two sentences, the conditions "homological dimension $\leqq 1$ ", "projective" and "hereditary" are replaced by "homological dimension $\leqq n "$, "homological dimension $<n$ ", and "right global dimension $\leqq n$ " for any positive integer $n$. The same is true of Corollary 3.2 through Theorem 3.4 (except, of course, that the projective modules of the constructions must still be assumed projective), if $S$ is left flat over all $R_{\alpha}$. This is needed so that the property "homological dimension $<n$ " will be preserved under extension of scalars, for $n>1$. However universal localizations (except for the special case of Ore localization with respect to a right denominator set, [13]) tend not to give left flat extensions, as the counterexamples of the next section will show.

REMARK 3.6. Can we not, on the basis of (58), add to Theorem

That $S$ would be semihereditary in all cases was asserted in [3], Theorem 6.4; but the argument was based on the false assumption that a direct limit of semihereditary rings was semihereditary; cf. Example 4.2 below. Of course, we now know the assertion to be true. 
3.4 another construction, "Forming full matrix rings $M_{n}(R)$, where $M_{n}(R)$ is made an $R$-ring by the diagonal map"? If we could, then for any hereditary ring $R$, the direct limit of $R \rightarrow M_{2}(R) \rightarrow M_{4}(R) \rightarrow \cdots$, which we may call $M_{2^{\infty}}(R)$, would also be hereditary. But for $R=$ $k[t]$ one has $M_{2^{\infty}}(R) \cong M_{2^{\infty}}(k)[t]$, which is easily shown not to be hereditary. The reason this argument fails is that, though $\Omega_{K}\left(M_{n}(R)\right)$ has a direct summand isomorphic to ${ }^{\otimes} \Omega_{K}(R)^{\otimes}$ as a bimodule, this is not the subbimodule generated by the image of $\Omega_{K}(R)$. So a direct limit such as $\Omega_{K}(R) \rightarrow \Omega_{K}\left(M_{2}(R)\right) \rightarrow \cdots$ will not be a big direct sum, as required to apply Corollary 3.2 .

REMARK 3.7. Tensor rings. If $K$ is an arbitrary ring, $B$ a $K$-bimodule, and $R=K\langle B\rangle$, then by (17) the exact sequence (61) becomes

$$
0 \longrightarrow{ }^{\otimes} B^{\otimes} \longrightarrow R \otimes_{K} R \longrightarrow R \longrightarrow 0 \text {. }
$$

Yu. V. Roganov uses this exact sequence to study the global dimension of $R$ in [23]. (His hypothesis that $B$, or in his notation $M$, be right projective may be weakened to "right flat" [16], p. 143.)

3.8. Lewin-Schreier formulas. If $R$ is a ring, let $\boldsymbol{K}_{0}(R)$ denote the universal enveloping group of the abelian semigroup of isomorphism classes of finitely generated projective right $R$-modules under $\oplus$. (The "Grothendieck group" of the category of finitely generated projective right $R$-modules [1].) The image of a projective module $P$ in $\boldsymbol{K}_{0}(R)$ will be denoted $[P]$. Now if an $R$-module $M$ has a resolution by finitely generated projective modules, $0 \rightarrow P_{n} \rightarrow \cdots$ $\rightarrow P_{0} \rightarrow M \rightarrow 0$, it is known that the element $\sum(-1)^{i}\left[P_{i}\right] \in \mathscr{K}_{0}(R)$ is an invariant of $M$, called its Euler characteristic $\chi_{R}(M)$.

If $R$ is a $K$-ring, then an $R$-module can also be looked at as a $K$-module, and it is natural to compare $\chi_{K}(M) \in K_{0}(K)$ and $\chi_{R}(M) \in$ $\boldsymbol{K}_{0}(R)$ when both are defined. J. Lewin [20] obtained a formula relating these two when $R$ is a free associative algebra over a field $K$, or a group algebra of a free group; he showed that this was a generalization of Schreier's formula relating the rank and index of a subgroup of a free group. Hence let us make the

(64) Definition. A $K$-ring $R$ will be called a right LewinSchreier K-ring if

(a) every right $R$-module $M$ which has a finite resolution by finitely generated projectives over $K$ also has such a resolution over $R$, and

(b) there exists a homomorphism $\lambda_{R, K}: \boldsymbol{K}_{0}(K) \rightarrow \boldsymbol{K}_{0}(R)$ such that 
for such an $M, \chi_{R}(M)=\lambda_{R, K} \chi_{K}(M)$.

We can use (62) to study this phenomenon. If $S$ is an $R$-ring we will denote by $\tau_{s, r}: \boldsymbol{K}_{0}(R) \rightarrow \boldsymbol{K}_{0}(S)$ the homomorphism induced by the functor $\otimes_{R} S$ on finitely generated projective right $R$-modules. If $S$ is left $R$-flat, then $\otimes_{k} S$ preserves resolutions, hence

If $S$ is a left-flat $R$-ring, then whenever an $R$-module $M$ has a finite resolution by finitely generated projectives, $M^{\otimes}$ has such a resolution as an $S$-module, and $\chi_{S}\left(M^{\otimes}\right)=\tau_{S, R} \chi_{R}(M)$.

From (62) and (17) we easily obtain

(66) Suppose $K$ is a division ring, $B$ a $K$-bimodule of finite right $K$-dimension $d$, and $R=K\langle B\rangle$. Then $R$ is a right LewinSchreier $K$-ring with $\lambda_{R, K}=(1-d) \tau_{R, K}$. (Cf. [13], pp. 85-86.)

Recall that for $K$ semisimple Artinian, a coproduct $S$ of faithful $K$-rings $R_{i}$ is right and left projective over each $R_{\imath}|2|$. In particular, it is left flat, so that (65) applies. If we take an $S$-module $M$, and compare the exact sequences obtained by applying (62) over the $R_{i}$ and over $S$, then these are related via (13), and we get

Suppose $K$ is a semisimple Artin ring, $R_{1}, \cdots, R_{n}$ are faithful $K$-rings, and $S=\Perp_{i} R_{i}$. Then if all $R_{i}$ are right LewinSchreier $K$-rings, so is $S$, and $\lambda_{S, K}=\left(\sum_{i} \tau_{S, R_{\imath}} \lambda_{R_{\tau_{\vartheta}, K}}\right)-$ $(n-1) \tau_{S, K}$. (Cf. [18], Theorem 8.)

Finally, if $S$ is a universal 2-sided localization of $R$ and $M$ an $S$-module, we can compare the exact sequences (62) coming from $S$ and $R$. In the sequence over $S$, the first term $M \otimes_{S}\left({ }^{\otimes} \Omega_{K}(R)^{\otimes}\right)$ simplifies to $M \otimes_{R} \Omega_{K}(R)^{\otimes}$. Now the Euler characteristic of this term can be obtained from that of $M \otimes_{R} \Omega_{K}(R)$ by $\tau_{S, n}$ if either the latter module is right projective (which will hold if $R$ is right hereditary) or $S$ is left $R$-flat (by (65)). We deduce:

(68) Let $K$ be a semisimple Artinian ring, $R$ a $K$-ring, and $S$ a universal 2-sided localization of $R$. Suppose either that $R$ is right hereditary, or that $S$ is left-flat over $R$. Then if $R$ is

a Lewin-Schreier $K$-ring, so is $S$, and $\lambda_{S, K}=\tau_{S, R} \lambda_{R, K}$.

In particular, from (66) and (68) one can recover Lewin's results on the Euler characteristic of finite $K$-dimensional modules over free $K$-algebras and group algebras of free groups [20]. Lewin has made essentially the same observation independently. 
We are not sure how widespread the "Lewin-Schreier" phenomenon is. One counterexample useful against naive conjectures is the $K$-algebra $K \times K=K\left\langle e \mid e^{2}=e\right\rangle$ ( $K$ a field) which is not LewinSchreier (it satisfies (64) (a) but not (b)). However, one might study relations between $\chi_{K}(M)$ and $\chi_{R}(M)$ of more general sorts than that one be a function of the other.

\section{Counterexamples and remarks.}

ExAmPLe 4.1. Global dimension $2 \mapsto \infty$. (Adapted from [18].) Let $K$ be a field, and form the $K$-algebras $R_{1}=K\langle x\rangle, R_{2}=$ $K\left\langle e, y, z \mid e^{2}=e\right\rangle . \quad R_{1}$ and $R_{2}$ are right (and left) hereditary by the results of [2], or Theorem 3.4 above. Let us map the polynomial ring $K[t]$ into $R_{1}$ by $t \mapsto x^{2}$, and into $R_{2}$ by $t \mapsto y e z$, and let $S=R_{1} \Perp_{K[t]} R_{2}=K\left\langle e, x, y, z \mid e^{2}=e, x^{2}=y e z\right\rangle$. Since $R_{1}$ and $R_{2}$ are faithfully flat (in fact, free) as left $K[t]$-modules, we can apply Corollary 7 and Theorem 9 (i) of [18] to conclude that

$$
\text { r.gl. } \begin{aligned}
\operatorname{dim} . S & \leqq \max \left(\text { r.gl. } \operatorname{dim} . R_{1}, \quad \text { r.gl. } \operatorname{dim} . R_{2}, 1+\text { r.gl.dim. } K[t]\right) \\
& =\max (1,1,2)=2 .
\end{aligned}
$$

On the other hand, because $e$ is idempotent, $S\left\langle(1-e)^{-1}\right\rangle=$ $S / S e S=K\left\langle x, y, z \mid x^{2}=0\right\rangle$, which has infinite right global dimension. (Since all these ring-constructions are right-left symmetric, these rings also have left global dimensions of the same values.)

Let us write $K\left\langle e \mid e^{2}=e\right\rangle$ as $K \times K$. Then as noted in [2], $S / S e S$ can be written $S \Perp_{K \times K}(K \times K) /(K \times\{0\})$, showing that the results of [2] on global dimensions of coproducts of faithful $R_{0}$-rings ( $R_{0}$ semisimple Artinian) do not go over to the nonfaithful case.

By Remark 3.5 above, $S\left\langle(1-e)^{-1}\right\rangle$ must be nonflat as a left $S$-module. And indeed, we see that $t=x^{2}$ is zero in this ring, hence this ring is a torsion module over $K[t]$, hence nonflat over $K[t]$, hence also over the left-flat $K[t]$-ring $S$.

Interestingly, no example is known where the global dimension shows a finite increment.

EXAMPLE 4.2. Loss of semiheredity. Let $K$ be a field, and consider the $K$-algebra $R$ presented by generators $x, y_{i}, z_{i}(i \geqq 0)$ and relations

$$
y_{i}=x y_{i+1}, \quad z_{i}=z_{i+1} x \quad(i \geqq 0) .
$$

Note that this is the direct limit of the algebras $R_{n}$ presented by similar generators and relations, but with the index $i$ restricted to be $\leqq n$. But it is easy to see that this presentation of $R_{n}$ allows 
us to eliminate all but three generators, and all relations, and write $R_{n}=K\left\langle x, y_{n}, z_{n}\right\rangle$. This is a free associative algebra, hence a fir (free ideal ring-see [13]. Some of the relevant definitions will be recalled in Remark 4.3 below). Hence the direct limit ring $R$ is, at least, a semifir ([13], Exercise 1.1.3, p.47) and so in particular, semihereditary. We remark that $R$ is the semigroup algebra $K U$ on the semigroup (with 1 ) $U$ presented by the same equations as $R$. It is easy to check from its "direct limit" description that $U$ can be identified with the subsemigroup of the free group on generators $x, y, z$ generated by the elements $x, y_{i}=x^{-i} y$, and $z_{i}=z x^{-i}$.

Now let $S$ denote the universal localization $R\left\langle y_{0}^{-1}\right\rangle$. This will also be a semigroup algebra $K U\left\langle y_{0}^{-1}\right\rangle$. But we should not be tempted to think that $U\left\langle y_{0}^{-1}\right\rangle$ will just be some larger subsemigroup of the free group on $x, y$, and $z$. The point is that when we universally invert $y_{0}=x^{i} y_{i}$, this does not lead to inverses of $x^{i}$ and $y_{i}$, but only to a right inverse, $y_{i} y_{0}^{-1}$, of $x^{i}$, and a left inverse, $y_{0}^{-1} x^{i}$, of $y_{i}$.

Now when an element $a$ of a ring $R$ has a right inverse $b$, then $b a-1$ is an idempotent, and $(b a-1) R$ is the right annihilator of $a$ in $R$. Hence in the above ring $S$, the chain of annihilators

$$
\text { rt. ann. }{ }_{s}(x) \subseteq \cdots \subseteq \text { rt. ann. }{ }_{s}\left(x^{i}\right) \subseteq \cdots
$$

takes the form

$$
\left(y_{1} y_{0}^{-1} x-1\right) S \leqq \cdots \leqq\left(y_{i} y_{0}^{-1} x^{i}-1\right) S \subseteq \cdots .
$$

To study (69) further, we must turn to the rings $S_{n}=R_{n}\left\langle y_{0}^{-1}\right\rangle=$ $K\left\langle x, y_{n}, z_{n}, u \mid u x^{n} y_{n}=1=x^{n} y_{n} u\right\rangle$, of which $S$ is the direct limit. A $K$-basis for $S_{n}$ is given by all words in $x, y_{n}, z_{n}$, and $u$ not involving either of the sequences $u x^{n} y_{n}$ or $x^{n} y_{n} u$. (For a general result on normal forms from which this follows immediately, see [7], Theorem 1.2.) From this it is easy to check that the $(i+1)$ st term of (69) does not annihilate $x^{i}$ in $S_{n}(n>i)$; i.e., $x^{i}\left(x^{n-i-1} y_{n} u x^{i+1}-1\right) \neq 0$; hence it does not annihilate $x^{i}$ in the limit-ring $S$, so (69) is strictly increasing. We also see that in $S_{n}, z_{n}$ is a non-zero-divisor, hence the right annihilator of $z_{0}=z_{n} x^{n}$ is the right annihilator of $x^{n}$, hence in $S$ the right annihilator of $z_{0}$ is the union of the right annihilators of the $x^{n}$. Since these form a strictly ascending chain, the right ideal $z_{0} S$ is not finitely related, hence not projective, hence $S$ is not right semihereditary, though it is a universal localization of a semifir.

REMARK 4.3. On direct limits of semihereditary rings. Note that the nonsemihereditary ring $S=R\left\langle y_{0}^{-1}\right\rangle$ constructed above is 
the direct limit of the rings $S_{n}=R_{n}\left\langle y_{0}^{-1}\right\rangle$, which by Theorem 3.3 are all hereditary. On the other hand we proved $R$ semihereditary using the fact that a special class of semihereditary rings, the semifirs, is closed under direct limits. The following remarks are intended to throw some light on the seemingly special behavior of this subclass.

Recall that in [4] a ring $R$ was named weakly semihereditary if whenever two maps of finitely generated projective right $R$-modules compose to 0:

$$
Q_{1} \stackrel{g}{\longrightarrow} P \stackrel{f}{\longrightarrow} Q_{2}
$$

there exists a direct sum decomposition $P=P_{1} \oplus P_{2}$ such that $f$ annihilates $P_{1}$, and $g$ has range in $P_{1}$; i.e., in terms of which the two maps have the "matrix" forms $\left(\begin{array}{l}* \\ 0\end{array}\right)$ and $\left(\begin{array}{l}0 \\ *\end{array}\right)$ respectively. (If we drop the condition that $Q_{1}$ be finitely generated, the above becomes the condition for $R$ to be right semihereditary.)

It is easy to see that

A direct limit of weakly semihereditary rings is weakly semihereditary.

We say that a ring $R$ has $A C C_{\oplus}$ on finitely generated projective modules if the semigroup of isomorphism classes of such modules has the property that there is no infinite chain of direct sum decompositions:

$$
P_{0}=P_{0}^{\prime} \oplus P_{1}, \cdots, P_{i}=P_{i}^{\prime} \oplus P_{i+1}, \cdots \quad\left(P_{i}^{\prime} \neq 0\right) .
$$

It was noted in [4] that a weakly semihereditary ring with $A C C_{\oplus}$ on finitely generated projective modules is right and left semihereditary. (The converse is not true.) Finally, note that

(71) The semigroup of isomorphism classes of finitely generated projective modules over a direct limit of rings is isomorphic to the direct limit of the corresponding semigroups for the given rings.

So if the given rings are semihereditary and if the above limit semigroup happens to have $A C C_{\oplus}$, the limit ring will again be semihereditary.

We now turn to semifirs. A semifir can be defined as a right semihereditary ring whose semigroup of isomorphism classes of finitely generated projective modules, with the additional structure of distinguished element given by the class of the free module of rank 1, is isomorphic to the semigroup of nonnegative integers with 
1 as distinguished element. When we form any direct limit of semifirs, we see by (71) that the semigroup of isomorphism classes of finitely generated projectives will still be the nonnegative integers. Since this has $A C C_{\oplus}$, the limit ring will also be semihereditary, and hence again a semifir.

(We also see that in the definition of a semifir, "right semihereditary" can be replaced by "weakly semihereditary". Cf. [13], p. 43, Theorem 1.1.1(f).)

In contrast, in the preceding example the idempotent elements $y_{i} y_{1}^{-1} x^{i}-1 \in R_{i}\left\langle y_{0}^{-1}\right\rangle$ led to a violation of $A C C_{\oplus}$ in the direct limit. A simpler example of a nonsemihereditary direct limit of hereditary rings is given by the system of commutative algebras over a field $K$ :

$$
K[t] \longrightarrow K \times K[t] \longrightarrow K \times K \times K[t] \longrightarrow \cdots
$$

where the $i+1$ st arrow carries $\left(a_{1}, \cdots, a_{i}, f(t)\right)$ to $\left(a_{1}, \cdots, a_{i}, f(0), f(t)\right)$. The image, in the limit ring, of $t \in$ the first ring, has annihilator which is not the annihilator of any idempotent element. (Cf. [4] Example 3.6.)

We conclude this discussion with the obvious

(72) Question: How does the property of being weakly semihereditary behave under the universal constructions treated in $\S 2$ ? In particular is it preserved under universal localizations?

In the commutative case, the characterization of weakly semihereditary commutative rings in [4], Proposition 6.2 leads to an affirmative answer to the latter question.

5. Doing without $K$. (This section, except Remark 5.4, assumes only a small part of what precedes: the first two paragraphs of $\S 1$, and the material of $\S 2.1$ through (4).)

We shall prove below that universal 2-sided localizations of right hereditary rings are right hereditary, without the assumption that our rings contain a semisimple subring $K$. Our proof is motivated by that of:

(73) Let $R \rightarrow S$ be an epimorphism of rings such that $S$ is flat as a left $R$-module. Then r.gl.dim. $S \leqq \mathrm{r}$.gl.dim. $R$. (Cf. [24], Cor. 1.3.)

Proof. If $M$ is a right $S$-module and $0 \rightarrow P_{n} \rightarrow \cdots \rightarrow P_{0} \rightarrow M \rightarrow 0$ is a projective resolution of $M$ as an $R$-module, then

$$
0 \longrightarrow P_{n} \otimes_{R} S \longrightarrow \cdots \longrightarrow P_{0} \otimes_{R} S \longrightarrow M \otimes_{R} S \longrightarrow 0
$$


is exact by left flatness of $S$, and

$$
M \otimes_{R} S \cong M
$$

because $R \rightarrow S$ is an epimorphism ([19], [24], [6], Theorem 18 (c)). Thus $h d_{S} M \leqq h d_{R} M$, and the conclusion follows.

(REMARK. The hypotheses of (73) are satisfied if $S$ is a localization of a commutative ring $R$, or more generally an "Ore localization" of an arbitrary ring $R$ with respect to a right denominator set [13].)

What we shall do in this section is describe a condition on a ring epimorphism $R \rightarrow S$ which is weaker than flatness, but will at least imply that if r.gl. $\operatorname{dim} . R$ is $\leqq 1$, then so is r.gl.dim. $S$. We shall then prove that this condition is (a) preserved by pushouts, and note easily that it is (b) respected by Morita equivalence and (c) possessed by the "canonical" example of universal localization:

$$
\left(\begin{array}{ll}
\boldsymbol{Z} & \boldsymbol{Z} \\
0 & \boldsymbol{Z}
\end{array}\right) \times \boldsymbol{Z} \subset\left(\begin{array}{ll}
\boldsymbol{Z} & \boldsymbol{Z} \\
\boldsymbol{Z} & \boldsymbol{Z}
\end{array}\right) \times \boldsymbol{Z}
$$

By the methods used in [3] we will deduce that it is possessed by all universal localizations, from which our desired result will follow.

To see the condition we want, note that while the proof of (73) used the full force of $R \rightarrow S$ being an epimorphism in arguing that for all right $S$-modules $M$, (75) holds, less than the full strength of flatness was used in proving (74) exact. Flatness says

$$
\text { For all right } R \text {-modules } M, \operatorname{Tor}_{1}^{R}(M, S)=0
$$

or equivalently

$$
\text { For all right } R \text {-modules } M \text { and all } i>0, \operatorname{Tor}_{i}^{R}(M, S)=0 .
$$

But the exactness of (74) only required (77) for $M$ a right $S$-module. For global dimension $\leqq 1$ we will in fact only need this for $i=1$.

To study this condition we need the following observation.

Let $R \rightarrow S$ be a ring homomorphism, $M$ a right $S$-module and $N$ a left $R$-module. Write $M$ as a surjective image of a projective right $S$-module $P$. Then the sequence

$$
\operatorname{Tor}_{1}^{R}(P, N) \longrightarrow \operatorname{Tor}_{1}^{R}(M, N) \longrightarrow \operatorname{Tor}_{1}^{S}\left(M, S \otimes_{R} N\right) \longrightarrow 0
$$

is exact, where the second map is the composition of natural maps

$$
\operatorname{Tor}_{1}^{R}(M, N) \longrightarrow \operatorname{Tor}_{1}^{R}\left(M, S \otimes_{R} N\right) \longrightarrow \operatorname{Tor}_{1}^{S}\left(M, S \otimes_{R} N\right) .
$$


This is [6] (7). Proof in brief: To an exact sequence $0 \rightarrow A \rightarrow P \rightarrow$ $M \rightarrow 0$, we apply $\operatorname{Tor}^{R}(-, N) \rightarrow \operatorname{Tor}^{S}\left(-, S \otimes_{R} N\right)$. The resulting map of long exact sequences gives isomorphisms on Tor ${ }_{0}$, while on Tor ${ }_{1}$, the second sequence has $P$-term 0 . Hence the map of $\operatorname{Tor}_{1}(M,-)$ 's, is in fact a cokernel as indicated in (78).

Proposition 5.1. For any ring epimorphism $R \rightarrow S$, the following conditions are equivalent:

(i ) The transformation Tor $_{1}^{R} \rightarrow$ Tor $_{1}^{S}$ of bifunctors of S-bimodules is an isomorphism.

(ii) For all right $S$-modules $M$ and left $R$-modules $N$, the map $\operatorname{Tor}_{1}^{R}(M, N) \rightarrow \operatorname{Tor}_{1}^{R}\left(M, S \otimes_{R} N\right)$ is surjective.

(iii) $\operatorname{Tor}_{1}^{R}(S, S)=0$.

(iv) For all right $S$-modules $M$, $\operatorname{Tor}_{1}^{R}(M, S)=0$ (cf. (76) above).

(ii*) and (iv*): The right-left duals of (ii) and (iv).

Proof. For any ring $\operatorname{map} R \rightarrow S$ we get (i) $\Rightarrow$ (ii) by noting that the composition (79) is surjective by (78), and applying (i) to its right-hand factor. We get (ii) $\Rightarrow$ (iii) by taking $M=S, N=R$ in (ii).

Now assume $R \rightarrow S$ an epimorphism. To get (iii) $\Rightarrow$ (iv) apply (78) with $M$ arbitrary, $P$ a free $S$-module, and $N=S$. Then (iii) makes the first term zero, while the epimorphism condition makes $S \otimes_{R} S=S$ in the third term, which is thus zero. Hence the middle term is zero. Finally, assuming (iv) we get (i) by applying the leftright dual of (78) to left and right $S$-modules $M, N$. Here $N$ is written as a homomorphic image of a free left module $Q$ so that the first term $\operatorname{Tor}_{1}^{R}(M, Q)$ becomes zero by (iv), so the second map is an isomorphism, and its codomain simplifies to $\operatorname{Tor}_{1}^{S}(M, N)$ by the epimorphism assumption. This completes the proof.

(80) Definition. A ring epimorphism $R \rightarrow S$ satisfying the equivalent conditions of Proposition 5.1 will be called a pseudoflat epimorphism.

(REMARK. The condition that $R \rightarrow S$ be an epimorphism has formulations analogous to (i)-(iv): (i') $\operatorname{Tor}_{0}^{R} \cong \operatorname{Tor}_{0}^{S}$, (ii') $\operatorname{Tor}_{0}^{R}(M, N) \rightarrow \operatorname{Tor}_{0}^{R}$ $(M, S \otimes N),\left(\mathrm{iii}^{\prime}\right) \operatorname{Tor}_{0}^{R}(S, S) \cong \operatorname{Tor}_{0}^{S}(S, S),\left(\mathrm{iv}^{\prime}\right) \operatorname{Tor}_{0}^{R}(M, S) \cong \operatorname{Tor}_{0}^{S}(M, S)$.)

We now record some easy consequences of Proposition 5.1. From (i) we get:

(81) A composition of pseudoflat ring epimorphisms is pseudoflat.

(82) The direct limit of a directed system of rings and pseudoflat epimorphisms is pseudoflat over each of the given rings. 
There is a partial converse to (81).

If a composition of ring homomorphisms $R \rightarrow S \rightarrow T$ is a pseudoflat epimorphism, and if $R \rightarrow S$ is an epimorphism, then the map $S \rightarrow T$ is a pseudoflat epimorphism.

To see this, note that for $R \rightarrow S$ any ring epimorphism, (78) applied to $S$-modules shows that $\operatorname{Tor}_{1}^{R} \rightarrow \operatorname{Tor}_{1}^{S}$ is surjective. (83) is easily deduced.

Recall that the $n \times n$ matrix ring functor $M_{n}$ induces equivalences of module categories (Morita equivalence), and these necessarily respect the construction Tor. Hence

A homomorphism $R \rightarrow S$ is a pseudoflat epimorphism if and only if $M_{n}(R) \rightarrow M_{n}(S)$ is.

From condition (iii) of Proposition 5.1 we see

If $R \rightarrow S$ is an epimorphism such that $S$ is either flat as a right $o r^{\circ}$ flat as a left $R$-module, then $R \rightarrow S$ is pseudoflat.

Finally, from (iv) and our earlier discussion we have

If $R \rightarrow S$ is a pseudofiat epimorphism, then for any right $S$-module $M,\left(\operatorname{hd}_{R} M \leqq 1\right) \Longrightarrow\left(h_{S} M \leqq 1\right)$. In particular, if $R$ is right hereditary, so is $S$.

(87) Example. A surjective homomorphism $R \rightarrow R / I$ will be a pseudoflat epimorphism if and only if $I^{2}=I$.

For $\operatorname{Tor}_{1}^{R}(R / I, R / I)=I / I^{2}$. Such an epimorphism need not be flat. For example if $R$ is a commutative integral domain, $R \rightarrow R / I$ is flat if and only if $I=0$ or $I=R$. But there are integral domains with nontrivial idempotent ideals, for instance any nonprincipal valuation ring. Cf. also Example 4.1 above.

We now want to prove that pushouts in the category of rings preserve pseudoflat epimorphisms. Let $K$ be a ring, let $R_{1}$ and $R_{2}$ be $K$-rings, and let $S$ be their coproduct as $K$-rings, $S=R_{1} \Perp_{K} R_{2}$, equivalently the pushout of the given pair of ring maps. Let us recall a characterization of the underlying $K$-bimodule of $S$ due to P.M. Cohn [12]:

Cohn obtains $S$ as the direct limit of a system of bimodule maps $W_{0} \rightarrow W_{1} \rightarrow \cdots$. Here $W_{n}$ represents, roughly, the elements of $S$ lying in the $n$-fold product $\cdots R_{1} R_{2} R_{1}$ in $S$. Precisely, we define $W_{0}=0, W_{1}=R_{1}$, and assume inductively that for some $n$ we have defined $K$-bimodules and maps, $W_{0} \rightarrow \cdots \rightarrow W_{n}(n \geqq 1)$, so that the 
odd terms are in fact $\left(R_{1}, K\right)$-bimodules, the even terms are $\left(R_{2}, K\right)$ bimodules, and the maps $W_{i} \rightarrow W_{i+2}$ respect these bimodule structures. Then $W_{n+1}$ is defined as the following pushout of $\left(R_{*}, K\right)$ bimodules, where $R_{*}$ denotes $R_{1}$ if $n+1$ is odd, $R_{2}$ if $n+1$ is even.

$$
R_{*} \otimes_{K} W_{n-1} \searrow_{R_{*} \otimes_{K} W_{n}}^{W_{n-1}}
$$

Here the upper solid arrow is induced by the left $R_{*}$-module structure of $W_{n-1}$, and the lower one by the map $W_{n-1} \rightarrow W_{n}$. We map $W_{n}$ into this pushout via its map into the bottom term of the above diagram.

Now taking $W_{\infty}=\operatorname{dir} . \lim W_{n}=\operatorname{dir} . \lim W_{2 m+1}=\operatorname{dir} . \lim W_{2 m}$, we see that this limit object has compatible left $K, R_{1}$, and $R_{2}$ module structures, hence is a left module over $S=R_{1} \Perp_{K} R_{2}$. Further, the map $W_{1}=R_{1} \rightarrow S$ is easily seen to extend to a left $S$-module map $W_{\infty} \rightarrow S$. Since this carries the left $S$-module generator 1 of $W_{\infty}$ to the free left module generator 1 of $S$, the map is an isomorphism, so $W_{\infty} \cong S$, as claimed. Using this description we can now prove

Proposition 5.2. Let $K$ be a ring, and $K \rightarrow L$ a pseudoflat ring epimorphism. Then for any $K$-ring $R$, the map $R \rightarrow S=R \Perp_{K} L$ is also a pseudoflat epimorphism.

Proof. Let us take $R_{1}=R, R_{2}=L$ in the preceding description. We observe that when $n=2 m-1$, so that $R_{*}=L$ in (88), the upper solid arrow of that diagram is an isomorphism, because $K \rightarrow L$ is an epimorphism. Hence the pushout is simply the other object in the diagram, i.e., $W_{2 m}=L \otimes_{K} W_{2 m-1}$. If we now substitute this into the diagram for $n=2 m$, we get a pushout of modules all described in terms of $W_{2 m-1}$ :

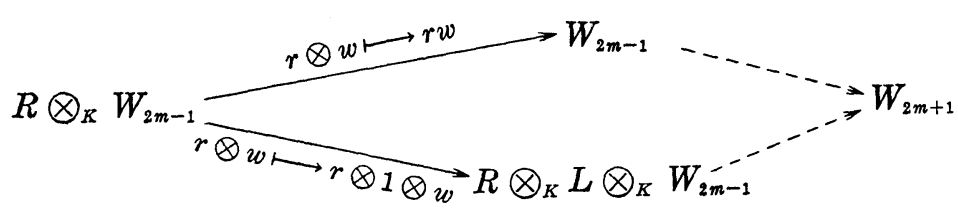

We shall now show that for all $m \geqq 1$, and all right $S$-modules $M$,

$$
\operatorname{Tor}_{1}^{R}\left(M, W_{2 m-1}\right)=0 \text {. }
$$

(In fact, (89) is equivalent to a description of $W_{2 m+1}$ as $U \otimes_{R} W_{2 m-1}$, where the $(R, R)$-bimodule $U$ is the pushout of the corresponding 
diagram with $R$ in place of $W_{2 m-1}$; and the arguments we shall give show more generally that for any right $S$-module $M$ and left $R$ module $N$, $\operatorname{Tor}_{1}^{R}(M, N)=0 \Rightarrow \operatorname{Tor}_{1}^{R}\left(M, U \otimes_{R} N\right)=0$.) We note that (90) is true for $m=1$, as $W_{1}=R$. Now for fixed $m$, assume (90). Let us write (89) as a presentation (91) of $W_{i_{m+1}}$, and then tensor to get a presentation (92) of $M \otimes_{R} W_{2 m+1}$ :

$$
\begin{aligned}
& R \otimes_{K} W_{2 m-1} \longrightarrow W_{2 m-1} \oplus\left(R \otimes_{K} L \otimes_{K} W_{2 m-1}\right) \longrightarrow W_{2 m+1} \longrightarrow 0 \\
& M \otimes_{K} W_{2 m-1} \longrightarrow\left(M \otimes_{R} W_{2 m-1}\right) \oplus\left(M \otimes_{K} L \otimes_{K} W_{2 m-1}\right)
\end{aligned}
$$$$
\longrightarrow M \otimes_{R} W_{2 m+1} \longrightarrow 0 \text {. }
$$

Since the $S$-module $M$ is in particular an $L$-module, and $K \rightarrow L$ is an epimorphism, the second summand in the middle object of (92) collapses to the left-hand object, so that in particular the lefthand map is $1-1$, and (92) with " $0 \rightarrow$ " on the left is a short exact sequence. (We can deduce more: this sequence will split, and $M \otimes_{R} W_{2 m-1} \cong M \otimes_{R} W_{2 m+1}$, but we do not see how to use this directly.) Now though (91) is not similarly "short exact", it is not hard to deduce from the short exactness of (92) that on applying $\operatorname{Tor}^{I 2}(M$, to (91) we get a surjection

(93) $\operatorname{Tor}_{1}^{R}\left(M, W_{2 m-1} \oplus\left(R \otimes_{K} L \otimes_{K} W_{2 m-1}\right)\right) \longrightarrow \operatorname{Tor}_{1}^{R}\left(M, W_{2 m+1}\right) \longrightarrow 0$.

By the hypothesis (90) we may delete the summand $W_{2 m-1}$ in the first Tor and still have a surjection.

Now consider the commutative diagram

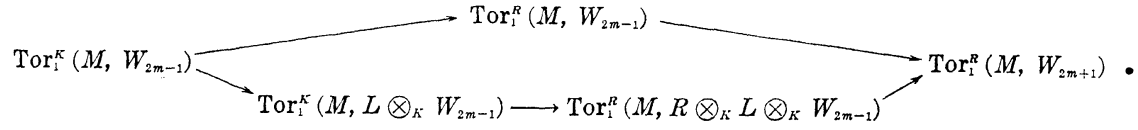

The term in the top branch is zero, so the composite map is zero. But in the bottom branch, the first arrow is surjective because $K \rightarrow L$ is pseudoflat (Proposition 5.1 (ii)), the second by (78), and the third by (93). Hence the right-most object must be 0 . This completes the inductive proof of (90) for all $\mathrm{m}$.

Since Tor commutes with direct limits, it follows that $\operatorname{Tor}_{1}^{R}(M, S)=0$. The map $R \rightarrow S$ is an epimorphism because pushouts preserve epimorphisms in any category. Hence by Proposition 5.1 (iv), this map is a pseudoflat epimorphism as claimed.

We may now deduce

Any universal 2-sided localization is a pseudoflat epimorphism. 
Indeed, by (82) (direct limits) we may reduce to the case where only a single morphism of projectives, $f: P \rightarrow Q$ is being inverted: $S=R\left\langle f^{-1}\right\rangle$. If we write $P \oplus Q$ as a direct summand in a free module ${ }^{n} R$, then by (84) (matrix rings) we may reduce to the case where $n=1$, so that $P=e_{P} R, Q=e_{Q} R$, where $e_{P}$, and $e_{Q}$ are orthogonal idempotents. We now regard $R$ as a $K$-ring where $K=\left(\begin{array}{cc}\boldsymbol{Z} & \boldsymbol{Z} \\ 0 & \boldsymbol{Z}\end{array}\right) \times \boldsymbol{Z}$, letting $\left(e_{11}, 0\right),\left(e_{22}, 0\right),\left(e_{12}, 0\right)$, and $(0,1)$ in $K$ go to $e_{\ell}, e_{P}, f$, and $1-e_{P}-e_{Q}$ respectively. Then universally inverting $f$ is equivalent to forming the pushout over $K$ of $R$ with $L=\left(\begin{array}{l}\boldsymbol{Z} \\ \boldsymbol{Z} \\ \boldsymbol{Z}\end{array}\right) \times \boldsymbol{Z}$. This epimorphic $K$-ring is pseudoflat by (85) because it is right flat. (Indeed, it is right projective: $L \cong\left(e_{11}, 1\right) K \oplus\left(e_{11}, 0\right) K$.) This gives (95). Hence by (86):

THeOREM 5.3. Any universal localization of a right hereditary ring is right hereditary.

We have not been able to obtain any general results on the structure of the semigroup of isomorphism classes of finitely generated projective modules over a universal localization. For examples of nontrivial behavior see [3] pp. 69-70, [8] § III; but for positive results under good hypotheses see [15] (which corrects and supersedes [14]).

It is amusing that (83) can be obtained as a corollary to Proposition 5.2. For from the hypothesis that $R \rightarrow S$ is an epimorphism, it follows that the pushout of the pseudoflat epimorphism $R \rightarrow T$ along $R \rightarrow S$ is $S \rightarrow T$.

Let us note

(96) Suppose $R \rightarrow S$ is a pseudoflat epimorphism.

(i) If $S$ is generated over $R$ by the inverses of a family $\Theta$ of morphisms of finitely generated projective $R$-modules, then the kernel of the map $R\left\langle\Theta^{-1}\right\rangle \rightarrow S$ is an idempotent ideal of $R\left\langle\Theta^{-1}\right\rangle$.

(ii) If $R$ is semihereditary (or more generally, $\operatorname{Tor}_{2}^{R}=0$ ) then the kernel of $R \rightarrow S$ is an idempotent ideal of $R$.

Indeed, (i) follows from (83) and (87). To see (ii), call the kernel $I$, and note that $\operatorname{Tor}_{2}^{R}=0$ makes $\operatorname{Tor}_{1}^{R}$ left exact in each variable, so $I / I^{2}=\operatorname{Tor}_{1}^{R}(R / I, R / I)$ embeds in $\operatorname{Tor}_{1}^{R}(S, S)$, which is 0 by pseudoflatness. The hypothesis of (ii) cannot be dropped, as is shown by the universal localization $K[s, t] /\left(s t, t^{2}\right) \rightarrow K\left[s, s^{-1}\right]$, which has nilpotent kernel.

REMARK 5.4. Relation between pseudoflat epimorphisms and 
universal derivations. To show this relation we need to do some calculating. Let $K$ be a ring, and $R \rightarrow S$ a map of $K$-rings. Then we have a commuting diagram of $(S, S)$-bimodules

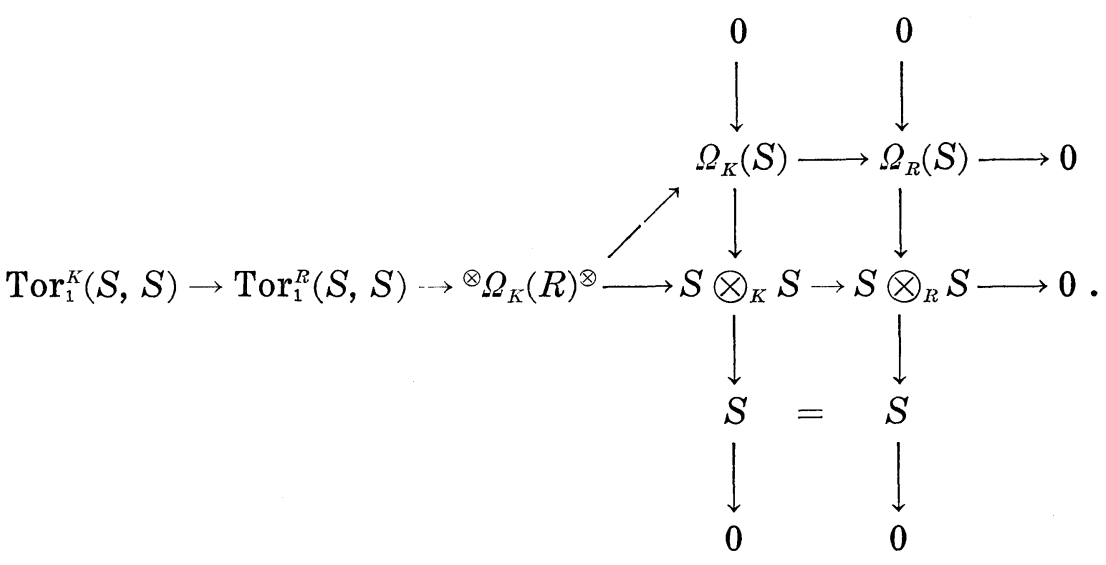

Here the long horizontal part is the exact sequence (8) of [6], and the vertical parts are (61). If we follow the upper branch on the right side of this diagram, while we note that by [6] (13) the cokernel of the leftmost arrow is $\operatorname{Tor}_{1}^{(R, K)}(S, S)$ (relative Tor) we obtain an exact sequence:

$$
0 \longrightarrow \operatorname{Tor}_{1}^{(R, K)}(S, S) \longrightarrow \Omega_{K}(R)^{\otimes} \longrightarrow \Omega_{K}(S) \longrightarrow \Omega_{R}(S) \longrightarrow 0 \text {. }
$$

Now the first stage in our derivations approach to universal localization was (4), which says that for $S$ a universal localization of $R$, the middle map of (98) is an isomorphism. By exactness this is equivalent to saying that the outer two terms are 0 . That the last term is 0 simply means that $R \rightarrow S$ is an epimorphism. Comparing the condition that the first term be zero with Proposition 5.1 (iii), we see that (4) is a " $K$-relativized" version of the statement that $R \rightarrow S$ is a pseudoflat epimorphism! If $S$ is right or left flat over $K$ so that the first term of (97) is zero, then $\operatorname{Tor}_{1}^{(R, K)}(S, S) \cong$ $\operatorname{Tor}_{1}^{P}(S, S)$, and (4) then says precisely that $R \rightarrow S$ is a pseudoflat epimorphism.

5.5. Possible generalizations. We observed earlier that the full strength of $R \rightarrow S$ being an epimorphism of rings was needed to make the assertion (75) that $M \otimes_{R} S \cong M$ in (74). But in fact one could adapt the argument there to a weaker hypothesis: that $M$ embed as a direct summand in the right $S$-module $M \otimes_{R} S$; in the terminology of relative homological algebra, that $M$ be projective relative to $R$. This holds, in particular, if $S$ is an $S$-bimodule direct summand in $S \otimes_{R} S$. We did not consider this condition be- 
cause of the difficulty of establishing equivalent conditions for pseudoflatness as in Proposition 5.1, without the epimorphism assumption. For similar reasons we did not study analogs of pseudoflatness with higher Tor $_{i}$ 's in place of Tor $_{1}$. However, someone might find it worthwhile to look further in these directions.

6. Appendix: universal localizations of $R$ and universal $R$ sfields-rectification of some past confusion. We have mentioned that the Ore localization of a ring $R$ with respect to a right or left denominator set [13] is a universal localization of $R$ in the sense of this paper. In particular, this applies to the sfield of fractions $K$ of a right or left Ore ring $R$.

However, such a sfield of fractions need not be a universal $R$-sfield in the sense of Cohn. (We are adapting Cohn's terminology to our own preferences by writing "sfield" for his (not necessarily commutative) "field". For results of Cohn's cited below, see [13, Ch. 7]. Cf. also [21], [17].) Let us describe the relation between these two conditions.

Let $R$ be a ring, $K$ an epic $R$-sfield, and let us write $\Sigma_{K}$ for the set of all square matrices (of all sizes) over $R$ whose images over $K$ are invertible. Thus, $R\left\langle\Sigma_{K}^{-1}\right\rangle$ is the "best approximation" to $K$ that we can get by starting with $R$ and universally inverting a set of square matrices. By results of Cohn, $R\left\langle\Sigma_{K}^{-1}\right\rangle$ is a local ring (possibly a sfield) having residue sfield canonically isomorphic to $K$. (One may deduce from this that we would have gotten the same ring if in trying to best approximate $K$ we had allowed iterated localizations, and the inversion of maps among nonfree projectives. Incidentally, Cohn writes $R_{\Sigma}$ for $R\left\langle\Sigma^{-1}\right\rangle_{\bullet}$ ) Now for two epic $R$-sfields $K$ and $L$ :

(99) The following conditions are equivalent (Cohn)

(i) $\Sigma_{K} \supseteq \Sigma_{L}$.

(ii) There exists a homomorphism of $R$-rings $R\left\langle\Sigma_{L}^{-1}\right\rangle \rightarrow$ $R\left\langle\Sigma_{K}^{-1}\right\rangle$.

(iii) There exists a specialization of $R$-sfields $K \rightarrow L$ (note reversed direction!)

(The reader not familiar with specializations of sfields may use (99) to replace statements about specializations by statements not using them in the latter part of this appendix.) (Cf. the case $R=\boldsymbol{Z}$, $K=\boldsymbol{Q}, L=\boldsymbol{Z} / p \boldsymbol{Z}$. Since a matrix over a commutative ring is inverted by inverting its determinant, one may replace $\Sigma_{K}$ by $\Sigma_{K} \cap R$ in considering this example.)

Cohn calls an epic $R$-sfield $K$ a universal $R$-sfield if the condi- 
tions of (99) hold for every other $R$-sfield $L . \quad R$ can have at most one universal $R$-sfield, up to isomorphism. (It need not have any.)

On the other hand, clearly $K$ will be a universal localization of $R$ as defined in this paper if and only if the $\operatorname{map} R\left\langle\Sigma_{K}^{-1}\right\rangle \rightarrow K$ is an isomorphism, i.e., if and only if $R\left\langle\Sigma_{K}^{-1}\right\rangle$ is a sfield.

The two straightforward relationships that exist between these conditions are

(100) The field of fractions $K$ of a commutative integral domain $R$ is both a universal $R$-sfield, and a universal localization of $R$.

(101) If $R$ has a universal $R$-sfield $K$, then $K$ is the only $R$-sfield (up to isomorphism) that can be a universal localization of $R$ (by (99) (ii)) though it may not be.

For very easy examples of how these conditions are not related, let $K$ and $L$ be fields, and consider the three commutative rings $R_{1}=K[\varepsilon] /\left(\varepsilon^{2}\right), R_{2}=K \times L, R_{3}=R_{1} \times L$. There is a unique epic $R_{1}$-sfield, $R_{1} /(\varepsilon)$, so this is a universal $R_{1}$-sfield, but since $R_{1}$ is already local, this field is not a universal localization of $R_{1}$. The two $R_{2}$-sfields $K$ and $L$ are both universal localizations, so neither can be a universal $R_{2}$-sfield by (101). $K$ and $L$ are also the two epic $R_{3}$-sfields. Although only one of them, $L$, is a universal localization, it is still not a universal $R_{3}$-sfield.

A ring $R$ may have a universal $R$-sfield $K$, but still have other epic $R$-sfields $L$ such that $R \rightarrow L$ is an embedding. For example, a free associative algebra $R=k\langle x, y, z\rangle$ has a universal $R$-sfield, but also embeds in the universal sfield of $k\langle x, u\rangle$ by $y \mapsto x u, z \mapsto x u^{2}$. In such cases, $L$ cannot be a universal localization of $R$ by (101), which means that when we localize $R$ at $\Sigma_{L}$, certain elements appear which go to zero in $L$, though no elements of $R$ do. (In the example noted, $y^{-1} z-x^{-1} y$ is such an element.)

On the other hand, examples are known of $R$-sfields $K$ such that $K$ is a universal localization of $R$, and $R$ embeds in $K$, but $K$ is not a universal $R$-sfield. Indeed, let us show that, as claimed above, there are Ore rings $R$ whose sfields of fractions $K$ are not universal $R$-sfields. One class of examples is noted in [13], Exercise 7.2 .11 (p.258). We shall sketch here a few more details than are shown there. Let $R_{0}$ be a ring with two nonisomorphic $R_{0}$-sfields $K$ and $L$ in which it embeds. Since they cannot each be specializations of the other, say there is no specialization (over $R_{0}$ ) from $K$ to $L$. Now adjoin a central indeterminate to $K$ to get a ring $K[t]$, and let $R$ be the subring thereof consisting of all elements with constant term in $R_{0}$. It is easy to verify that, like $K[t], R$ is right 
and left Ore and has sfield of fractions $K(t)$. But if we make $L$ an $R$-sfield by $R \stackrel{t \mapsto 0}{\longrightarrow} R_{0} \rightarrow L$, then $K(t)$ cannot be specialized to $L$ because $K$ cannot, so $K(t)$ is not a universal $R$-sfield. If in fact $L$ was a universal localization of $R_{0}$, then one may easily show that as an $R$-sfield it is also a universal localization.

Another example, in which $R$ has polynomial identity, is noted in [5] Exercise 12.2. Let us describe here a variant in which $R$ is also Noetherian. Let $\boldsymbol{C}$ and $\boldsymbol{H}$ denote the sfields of complex numbers and quaternions, and $\omega \in C$ a primitive cube root of unity. Let $R^{\prime}$ denote the $C$-algebra presented by generators $X, Y$ and the relation $Y X=\omega X Y$. Define a homomorphism $f$ from $R^{\prime}$ to the matrix ring $M_{3}(\boldsymbol{C})$ by

$$
f(X)=\left(\begin{array}{ccc}
\omega & 0 & 0 \\
0 & \omega^{2} & 0 \\
0 & 0 & 1
\end{array}\right) \quad f(Y)=\left(\begin{array}{lll}
0 & 1 & 0 \\
0 & 0 & 1 \\
1 & 0 & 0
\end{array}\right)
$$

One easily verifies that $f$ is well-defined. It is also surjective because $e_{33}=\left(f(X)^{2}+f(X)+1\right) / 3$ and $e_{i j}=f(Y)^{3-i} e_{33} f(Y)^{j}$.

Now let us consider the quaternion sfield $\boldsymbol{H}$ to be the sub- $\boldsymbol{R}$ algebra of $M_{2}(\boldsymbol{C})$ generated by $\boldsymbol{i}=i e_{11}-i e_{22}$ and $\boldsymbol{j}=e_{12}-e_{21}$. Then $M_{3}(\boldsymbol{C})$ contains $\left(\begin{array}{ll}\boldsymbol{H} & 0 \\ 0 & \boldsymbol{C}\end{array}\right)$, and so we may define $R=f^{-1}\left(\begin{array}{ll}\boldsymbol{H} & 0 \\ 0 & \boldsymbol{C}\end{array}\right) \subseteq R^{\prime}$.

One easily verifies that $R^{\prime}$, and hence also its sub-R-algebra of finite codimension $R$, is a Noetherian domain with polynomial identity whose sfield of fractions $K$ has dimension $9=3^{2}$ over its center, $C\left(X^{3}, Y^{3}\right)$. Hence in the language of [5], $R, R^{\prime}$, and $K$ have p.i. degree 3. Also, by construction we have surjective homomorphisms $R \rightarrow \boldsymbol{H} \times \boldsymbol{C} \rightarrow \boldsymbol{H}$. But the $R$-sfield $K$, of p.i. degree 3 , cannot be specialized onto the $R$-sfield $\boldsymbol{H}$ of p.i. degree 2 because 2 is not a divisor of 3 ([9], Cor. 6.9). So $K$ is not a universal $R$-sfield.

We have dwelt on these points because the temptation to confuse these two types of universality is great, having led Cohn, for instance, to assume in [13], (p.254, Example 2) that the sfield of fractions of a Noetherian domain $R$ is a universal $R$-sfield, and the first author of this paper to assert in the opposite direction (in [3], p. 70, 9th line from bottom, and various personal communications) that Ore localizations were not generally universal localizations!

Let us record for reference an explicit example of a matrix over the p.i. ring $R$ constructed above, which is singular over $R$, and hence over its sfield of fractions $K$, but is nonsingular over the $R$-sfield $\boldsymbol{H}$. Let $c=Y^{3}-Y^{6}$ and $d=1-X^{3}$. These are central in $R^{\prime}$, and their images in $M_{3}(\boldsymbol{C})$ are zero so that $c R^{\prime}+d R^{\prime}$ lies in $R$ and has image zero in $\boldsymbol{H}$. Let $u=(1-X)^{2}, \quad v=$ 
$(1-X)\left(Y^{2}-Y^{3}-Y^{4}\right)(1-X), w=(1-X)\left(Y-Y^{2}\right)(1-X) . \quad$ One checks that these elements lie in $R$, and have nonzero image in $\boldsymbol{H}$. Finally, let $p=\left(1+X+X^{2}\right)\left(Y^{2}+Y^{3}+Y^{4}\right)(1-X), r=2(1-X) Y^{2}(1-X)$. These elements will not lie in $R$; but we note that $u p=(r-v) d$, $w p=u c d$, and that $r^{2}$ is also a multiple of $d$ in $R^{\prime}$ (because it has left factors $\left.(1-X),(1-\omega X),\left(1-\omega^{2} X\right)\right)$. So all three of these products lie in $R$ and have image zero in $\boldsymbol{H}$. The above equations immediately yield the singularity result in $R$

$$
\left(\begin{array}{cccc}
r^{2}-v^{2} & u & 0 & v u \\
u v c & w & 0 & -u^{2} c \\
u v c & 0 & w & 0 \\
u c & 0 & 0 & w
\end{array}\right)\left(\begin{array}{c}
-d^{2} \\
p r d \\
p v d \\
p d
\end{array}\right)=0 .
$$

But because $c$ and $r^{2}$ go to zero in $\boldsymbol{H}$ but $v$ and $w$ do not, the above $4 \times 4$ matrix has nonsingular image over $\boldsymbol{H}$.)

6.1. (Added in proof.) Let us partially order the isomorphism classes of epic $R$-sfields by writing $K<L$ when the equivalent conditions of (99) are satisfied, and let us call an epic $R$-sfield $K$ "weakly universal" if it is a minimal element in this partially ordered set. (If $R$ is commutative, for instance, weakly universal $R$-sfields correspond to minimal prime ideals of of $R$.) One can show that every chain of $R$-sfields has a lower bound in this partially ordered set, hence by Zorn's Lemma, every epic $R$-sfield is a specialization of at least one weakly universal $R$-sfield. In particular, if $R$ has any $R$ sfields it has weakly universal ones, and an $R$-sfield $K$ is universal if and only if it is the unique weakly universal $R$-sfield.

It is now easy to see that the proper generalization of (101) (which holds for the same reasons) is: The $R$-sfields which are universal localizations of $R$ form a subset, possibly proper, of the weakly universal $R$-sfields.

The paragraph following (101) gave easy examples of a ring $R$ having more than one $R$-sfields which were universal localizations, and a ring $R$ having a universal $R$-sfield which was not a universal localization. We remarked in the preprint version of this paper that we did not know examples with these properties such that $R$ actually embedded in the sfield(s) in question. The first author has found such examples. We shall sketch the first (embeddability in more than one sfield which is a universal localization) below. The other example is at present quite complicated, and we will not give it here.

Let $k$ be a field, and $R$ the $k$-algebra having a universal $3 \times 3$ idempotent matrix $A$. Then $R$-sfields $K$ may be classified by the 
rank $i$ of the image of $A$. It turns out that $R$ has precisely 4 weakly universal $R$-sfields $K_{i}(i=0,1,2,3)$ each universal for $R$ sfields over which $A$ has rank $i$. One finds that each $K_{i}$ is a universal localization of $R$, and that $R$ embeds in both $K_{1}$ and $K_{2}$.

(Some details: If to $R$ we adjoin a universal inverse to $a_{13}$, we find that $A$ becomes similar to $\left(\begin{array}{lll}1 & 0 & 0 \\ 0 & e & 0 \\ 0 & 0 & 0\end{array}\right)$, where $e=a_{22}-a_{23} a_{13}^{-1} a_{32}$; and $R\left\langle a_{13}^{-1}\right\rangle$ can be written $k\left\langle a_{13}, a_{13}^{-1}, a_{12}, a_{23}, a_{33}, e \mid e^{2}=e\right\rangle$. By now inverting $1-e$ (respectively $e$ ) we get a free algebra with one generator inverted, $S_{1}$ (resp. $\left.S_{2}\right) \cong k\left\langle a_{13}, a_{13}^{-1}, a_{12}, a_{23}, a_{33}\right\rangle$. Such an algebra is known to have a universal sfield which is a universal localization, and in which it embeds. $R$ is shown by normal form arguments to map injectively into $S_{1}\left(\operatorname{resp} . S_{\mathrm{s}}\right.$ ).)

(That $R$ has precisely 4 weakly universal sfields, as described, is not necessary to the example per se but may be verifield by applying [3] to show $R$ hereditary and determine its semigroup of finitely generated projectives, then using [25] Theorem 23. If one wishes to modify this example to eliminate the two weakly universal $R$-sfields $K_{0}, K_{3}$ in which $R$ does not embed, one may achieve this by throwing in two more generators, $p, q$, and the relation $p a_{13}+a_{13} q=1$. This insures that $a_{13}$ does not go to zero under any homomorphism into a field, but avoids the loss of injectivity at $K_{1}$ and $K_{2}$ that would result if we were to do this by simply replacing $R$ by $R\left\langle a_{13}^{-1}\right\rangle$.)

7. Appendix. A simplifying framework for derivations and projective modules. Let $R$ be a ring, $\delta: R \rightarrow B$ a derivation into a bimodule, and $u: E^{m} R \rightarrow F^{n} R$ a morphism of finitely generated projective modules, with matrix $U$. Recall that if we decompose $U \in{ }^{n} R^{m}$ and $\delta U \in{ }^{n} B^{m}$ into their $(F, E)-,\left(F, E^{\prime}\right)-,\left(F, E^{\prime}\right)-$, and $\left(F^{\prime}, E^{\prime}\right)-$ components, the resulting decompositions will have the forms

$$
U=\left(\begin{array}{ll}
U & 0 \\
0 & 0
\end{array}\right), \quad \delta U=\left(\begin{array}{ll}
F(\delta U) E & U(\delta E) \\
(\delta F) U & 0
\end{array}\right) \quad \text { (cf. (31), (36)) . }
$$

Now considering $E, F, \delta E, \delta F$, and $U$ as "given", this means that though $\delta U$ is not itself an $(F, E)$-matrix, the "new information" that it brings is contained in the $(F, E)$-matrix $F(\delta U) E=U$ $(\delta F) U-U(\delta E)$ (which is the reason for (30), (31)). This suggests that the operation $U \mapsto F(\delta U) E$ might be worth considering in place of $\delta$ itself. Since the description of this operation requires that the matrix $U$ be given together with idempotents $E$ and $F$ indicating its intended "domain" and "codomain", this requires a categorytheoretic approach. But we must recall what we noted in the last 
paragraph of $\S 1$, that even for free modules, there is no invariant concept of applying a derivation $\delta$ to a module morphism, until "coordinates" are chosen for the modules. This leads to the following formalism: (We shall leave out the "k-structure" introduced in $\S 2.7$ here to allow us to concentrate on what is essential.)

If $R$ is a ring, then we define the category of coordinatized finitely generated projective right $R$-modules, denoted $/ R /$, to have for objects all idempotent square matrices $E$ over $R$, and for morphisms all 3 -tuples $(F, U, E)$ such that $F, E \in \mathrm{Ob} / R /$ and $U$ is an $(F, E)$-matrix over $R$. The domain and codomain of $(F, U, E)$ are, of course, $E$ and $F$ respectively, and composition is given by $(F, U, E) \circ(E, V, D)=(F, U V, D)$. Note that $/ R /$ has a natural additive structure, and is equivalent to the additive category of all finitely generated projective right $R$-modules.

If $\mathscr{A}$ and $\mathscr{A}$ ' are $\mathscr{A} l$-categories (in earlier usage called "preadditive categories") then in the spirit of [22] one may define an $\left(\mathscr{A}, \mathscr{A}^{\prime}\right)$-bimodule to mean a functor $\mathscr{B}: \mathscr{A}^{\mathrm{op}} \times \mathscr{A}^{\prime} \rightarrow \mathscr{A} \mathscr{C}$ (the category of abelian groups). Concretely, this means a function associating to each pair of objects, $X \in \mathrm{Ob} \mathscr{A}, X^{\prime} \in \mathrm{Ob} \mathscr{\mathscr { A } ^ { \prime }}$, an abelian group $\mathscr{B}\left(X, X^{\prime}\right)$, so that morphisms of $\mathscr{A}, f: Y \rightarrow X$, carry elements $b \in \mathscr{B}\left(X, X^{\prime}\right)$ to elements $b f \in \mathscr{B}\left(Y, X^{\prime}\right)$ and morphisms $g: X^{\prime} \rightarrow Y^{\prime}$ of $\mathscr{A}^{\prime}$ similarly act "on the left" to carry $\mathscr{B}\left(X, X^{\prime}\right)$ into $\mathscr{B}\left(X, Y^{\prime}\right)$, and these actions satisfy the associative law. (It might seem more natural to write $\mathscr{B}\left(X^{\prime}, X\right)$ for $\mathscr{B}\left(X, X^{\prime}\right)$ so that the object affected by left and right actions appear on the left and right respectively. We have made the opposite choice here for conformity with the notation for Hom-sets, in which we are following the traditional order: $\mathscr{A}(X, Y)=\{\mathscr{A}$-morphisms from $X$ to $Y\}$. Thus an $\mathscr{A}$ category, like a ring, becomes a bimodule over itself.)

Now if $R$ is a ring, and $B$ an $R$-bimodule in the usual sense, we can define an $/ R /$-bimodule $/ B /$ by taking $/ B /(E, F)=\{(F, E)$ matrices in $\left.{ }^{n} B^{m}\right\}$. Multiplication of matrices over $B$ by matrices over $R$ gives the bimodule structure.

If $\delta: R \rightarrow B$ is a derivation, then for every $u=(F, U, E) \in$ $\mid R /(E, F)$ let us define $\delta u=(F, F(\delta U) E, E) \in / B /(E, F)$. This operation clearly respects addition. Furthermore, given $u$ as above and $v=(E, V, D) \in / R /(D, E)$, we find

$$
\begin{aligned}
\delta(u v) & =(F, F(\delta(U V)) D, D)=(F, F(\delta U) V D+F U(\delta V) D, D) \\
& =(F, F(\delta U) E \cdot V, D)+(F, U \cdot E(\delta V) D, D) \\
& =(\delta u) v+u(\delta v)
\end{aligned}
$$

where the next-to-last step uses the conditions $V D=V=E V$, $F U=U=U E$. It is natural to call an operation $\delta$ with the above 
properties a derivation from the $\mathscr{A}$ a-category $\mid R /$ to the $/ R /$ bimodule $\mid B /$.

This formalism makes many of the computations we did in $\S 2$ much simpler. Thus, equation (9), for the action of $d$ on the 2 -sided inverse $u$ of a morphism $a$, becomes

$$
d u=-u(d a) u,
$$

exactly like (8). Similarly, (29) simply says that when $S=$ $R\left\langle u: P^{\otimes} \rightarrow Q^{\otimes}\right\rangle$, then $\Omega_{K}(S)$ is generated over ${ }^{\otimes} \Omega_{K}(R)^{\otimes}$ by the entries of $d u \in / \Omega_{K}(S) /(F, E)$, subject to no relations, beyond those defining an $(F, E)$-element. The one complication is that a given matrix $U$ will behave differently under $d$ depending on which $/ R /$-Homset one regards it in. For example, if $H$ is an idempotent $(F, F)$ matrix, (i.e., $H^{n} R$ a direct summand in the projective $R$-module $\left.F^{n} R\right)$ then an $(H, E)$-matrix $U$ is also an $(F, E)$-matrix. However, it is easy to relate the two ways of looking at $U$ : if we write $u=(H, U, E)$, and $i=(F, H, H)$ representing the inclusion of the summand $H$ (the module $H^{n} R$ ) in $F$ (the module $F^{n} R$ ), then $(F, U, E)=i u$; and the relation between the derivatives of these two manifestations of $U$ is given by $d(i u)=(d i) u+i(d u)$.

If $R \rightarrow S$ is a ring homomorphism, then on the object set of $|R|$ one can form a new category $/\left.S\right|_{R-p r o j}$, in which the morphisms from $E$ to $F$ are the $(F, E)$-matrices over $S$ (where $E$ and $F$ act via their images in $S$ ). This corresponds to looking at the category of those projective $S$-modules $P^{\otimes}$ induced by projective $R$-modules. The larger category /S/ can be constructed (up to a natural equivalence) from $/\left.S\right|_{R-\text { proj }}$ by taking for objects all idempotent endomorphisms $e$ of objects of $/ S /_{n-\text { pros }}$, and for morphisms between such objects the triples $(f, u, e)$ with $u=f u e$. Given a commuting diagram of rings and derivations

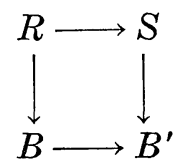

we get a commuting diagram of categories

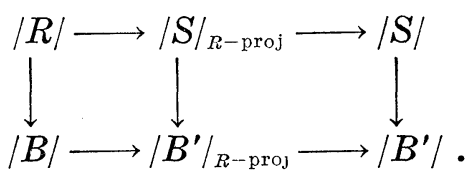

This allows us to study ring constructions in which new direct-sum decompositions $P^{\otimes}=P_{1} \oplus P_{2}$ appear (e.g., $R\left\langle u: P^{\otimes} \rightarrow P^{\otimes} \mid u^{2}=u\right\rangle$, $R\left\langle u: Q^{\otimes} \rightarrow P^{\otimes} \mid a u=1_{Q}\right\rangle$ as in (37) and (46)) without having to go 
back and refer to the way $P$ itself was a summand of a free module.

Some observations on the "disturbing" fact that the property $d u=0$ is not respected by isomorphism in $/ R /$. If we recall that derivations originated in physics with the study of velocity, we see that this is analogous to the phenomenon that a particle motionless in one frame may be moving in another, and if rotating coordinates are allowed even the property that two particles have the same velocity is not invariant. Given two coordinate systems rotating with respect to one another, we can at any instant transform position coordinates from one to another in a well-defined manner, but not velocities. We can, however, translate pairs: (position, velocity) from one frame to the other.

Likewise in our context, though "o $u$ " cannot be treated in an invariant manner, the pair $(u, \hat{\delta} u)$ can: Given isomorphisms in $/ R /$, $i: E^{\prime} \cong E, j: F \cong F^{\prime}$ we get an isomorphism $/ R /(E, F) \times / B /(E, F) \cong$ $/ R /\left(E^{\prime}, F^{\prime}\right) \times / B /\left(E^{\prime}, F^{\prime}\right)$ under which pairs $(u, \delta u)$ go to pairs $\left(u^{\prime}, \delta u^{\prime}\right)$, namely $(r, b) \leftrightarrow(j r i,(\delta j) r i+j b i+j r(\delta i))$. It is because the second component does not depend on $b$ alone that $\delta u$ alone cannot be so treated!

An interesting case. Let $\delta: R \rightarrow B$ be a derivation, and $E \in{ }^{n} R^{n}$ an idempotent. In general, no conjugate $A^{-1} E A$ need be annihilated by $\hat{o}$. But one can always find an object $F \in / R /$ isomorphic to $I_{n}$, on which the idempotent corresponding to $E$ is killed by $\delta$, namely the $2 n \times 2 n$ matrix $\left(\begin{array}{cc}E & 0 \\ 0 & I-E\end{array}\right)$, the "external" direct sum of the objects $E$ and $I-E$.

\section{REFERENCES}

1. H. Bass, Algebraic K-Theory, Benjamin, N. Y., 1968.

2. G. M. Bergman, Modules over coproducts of rings, Trans. Amer. Math. Soc., 200 (1974), 1-32.

3. Coproducts and some universal ring constructions, Trans. Amer. Math. Soc., 200 (1974), 33-88.

4. - Hereditarily and cohereditarily projective modules, pp. 29-62 of Ring Theory (proceedings of a conference at Park City, Utah, March 1971, R. Gordon ed.), Academic Press, N. Y., 1972.

5. - Rational relations and rational identities in division rings, $I$ and $I I$, J. Algebra 43 (1976), 252-266 and 267-297.

6. G. M. Bergman and W. Dicks, On universal derivations, J. Algebra, 36 (1975), 193-211.

7. G. M. Bergman, The diamond lemma for ring theory, Advances in Math., 26 (1978), 178-218.

8. - Some examples in PI ring theory, Israel J. Math., 18 (1974), 257-277.

9. G. M. Bergman and L. W. Small, P.i. degrees and prime ideals, J. Algebra, 33 (1975), 435-462.

10. N. Bourbaki, Algèbre Ch. III (=Éléments de Mathématique 1:2:3), Hermann, Paris, 
1970. (Material we cite is not in earlier editions.)

11. H. Cartan and S. Eilenberg, Homological algebra, Princeton, N. J., 1956.

12. P. M. Cohn, On the free product of associative rings, Math. Zeits., 71 (1959), 380-398.

13. P. M. Cohn, Free Rings and Their Relations, Academic Press, London, N. Y., 1971.

14. - Localization in semifirs, Bull. London Math. Soc., 6 (1974), 13-20.

15. P. M. Cohn and W. Dicks, Localization in semifirs, II, J. London Math. Soc., (2) 13 (1976), 411-418.

16. P. M. Cohn, Algebra II, John Wiley and Sons, (1977).

17. - Skew Field Constructions, London Math. Soc. Lecture Note Series 27, Cambridge University Press, 1977.

18. W. Dicks, Mayer-Vietoris presentations over colimits of rings, Proc. London Math. Soc., (3) 34 (1977), 557-576.

19. J. T. Knight, On epimorphisms of noncommutative rings, Proc. Cambridge Phil. Soc., 68 (1970), 589-600.

20. J. Lewin, Free modules over free algebras and free group algebras: the Schreier technique, Trans. Amer. Math. Soc., 145 (1969), 455-465.

21. P. Malcolmson, A prime matrix ideal yields a skew field, J. London Math. Soc., (to appear).

22. B. Mitchell, Rings with several objects, Advances in Math., 8 (1972), 1-161.

23. Үu. V. Roganov, Ю. В. Роганов, Гомологическая размерность тензорной алгебры проективного бимодуля, (Homological dimension of the tensor algebra of a projective bimodule. Russian.) Mat. Zametki, 18 (1975), 895-902.

24. L. Silver, Noncommutative localization and applications, J. Algebra, 7 (1967), 44-76.

25. W. Dicks and E. D. Sontag, Sylvester domains, J. Pure and Applied Algebra, 13 (1978), 243-275.

Received January 12, 1977.

University of California

Berkeley, CA 94720

AND

BEDFORD COLLEGE

LONDON NW1 4NS

ENGLAND 



\section{PACIFIC JOURNAL OF MATHEMATICS}

\section{EDITORS}

RichaRd ARENS (Managing Editor)

University of California

Los Angeles, CA 90024

Charles W. Curtis

University of Oregon

Eugene, OR 97403

C. C. Moore

University of California

Berkeley, CA 94720

\section{J. DUGUNDJI}

Department of Mathematics

University of Southern California

Los Angeles, CA 90007

R. FinN and J. Milgram

Stanford University

Stanford, CA 94305

\section{E. F. BECKENBACH \\ B. H. NeumanN

\author{
UNIVERSITY OF BRITISH COLUMBIA \\ CALIFORNIA INSTITUTE OF TECHNOLOGY \\ UNIVERSITY OF CALIFORNIA \\ MONTANA STATE UNIVERSITY \\ UNIVERSITY OF NEVADA, RENO \\ NEW MEXICO STATE UNIVERSITY \\ OREGON STATE UNIVERSITY \\ UNIVERSITY OF OREGON
}

F. WOLF

K. Yoshida

\section{SUPPORTING INSTITUTIONS}

\author{
UNIVERSITY OF SOUTHERN CALIFORNIA \\ STANFORD UNIVERSITY \\ UNIVERSITY OF HAWAII \\ UNIVERSITY OF TOKYO \\ UNIVERSITY OF UTAH \\ WASHINGTON STATE UNIVERSITY \\ UNIVERSITY OF WASHINGTON
}

The Supporting Institutions listed above contribute to the cost of publication of this Journal, but they are not owners or publishers and have no responsibility for its content or policies.

Mathematical papers intended for publication in the Pacific Journal of Mathematics should be in typed form or offset-reproduced, (not dittoed), double spaced with large margins. Please do not use built up fractions in the text of the manuscript. However, you may use them in the displayed equations. Underline Greek letters in red, German in green, and script in blue. The first paragraph or two must be capable of being used separately as a synopsis of the entire paper. Items of the bibliography should not be cited there unless absolutely necessary, in which case they must be identified by author and journal, rather than by item number. Manuscripts, in triplicate, may be sent to any one of the editors. Please classify according to the scheme of Math. Reviews, Index to Vol. 39. All other communications should be addressed to the managing editor, or Elaine Barth, University of California, Los Angeles, California, 90024.

50 reprints to each author are provided free for each article, only if page charges have been substantially paid. Additional copies may be obtained at cost in multiples of 50 .

The Pacific Journal of Mathematics is issued monthly as of January 1966. Regular subscription rate: $\$ 72.00$ a year (6 Vols., 12 issues). Special rate: $\$ 36.00$ a year to individual members of supporting institutions.

Subscriptions, orders for numbers issued in the last three calendar years, and changes of address should be sent to Pacific Journal of Mathematics, P.O. Box 969, Carmel Valley, CA 93924, U.S.A. Older back numbers obtainable from Kraus Periodicals Co., Route 100, Millwood, NY 10546.

PUBLISHED BY PACIFIC JOURNAL OF MATHEMATICS, A NON-PROFIT CORPORATION

Printed at Kokusai Bunken Insatsusha (International Academic Printing Co., Ltd.). 8-8, 3-chome, Takadanobaba, Shinjuku-ku, Tokyo 160, Japan. 


\section{Pacific Journal of Mathematics \\ Vol. 79, No. $2 \quad$ June, 1978}

David R. Adams, Quasi-additivity and sets of finite $L^{p}$-capacity ........ 283

George M. Bergman and Warren Dicks, Universal derivations and universal

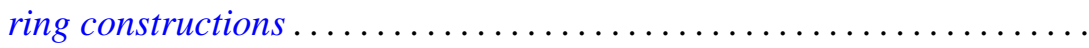

Robert F. Brown, Addendum to: "Fixed points of automorphisms of compact Lie groups".........................................

Eugene Frank Cornelius, Jr., Characterization of a class of torsion free

groups in terms of endomorphisms .......................

Andres del Junco, A simple measure-preserving transformation with trivial centralizer..................................... 357

Allan Lee Edmonds, Extending a branched covering over a handle ...... 363

Sjur Flam, A characterizaton of $\mathbf{R}^{2}$ by the concept of mild convexity .......

Claus Gerhardt, $L^{p}$-estimates for solutions to the instationary Navier-Stokes

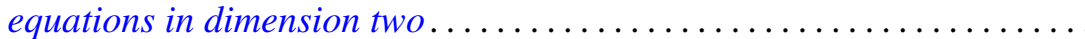

Kensaku Gomi, Finite groups with a standard subgroup isomorphic to

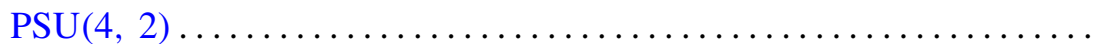

E. E. Guerin, A convolution related to Golomb's root function ........... 463

$\mathrm{H}$. B. Hamilton, Modularity of the congruence lattice of a commutative cancellative semigroup .................................

Stephen J. Haris, Complete reducibility of admissible representations over function fields.

Shigeru Itoh and Wataru Takahashi, The common fixed point theory of singlevalued mappings and multivalued mappings... ...

James E. Joseph, Multifunctions and graphs .............

Bruce Magurn, Images of $S K_{1} Z G$. .

Arnold Koster Pizer, A note on a conjecture of Hecke... .

Marlon C. Rayburn, Maps and h-normal spaces ...........

Barada K. Ray and Billy E. Rhoades, Corrections to: "Fixed-point theorems for mappings with a contractive iterate" ..............

Charles Irvin Vinsonhaler, Corrections to: "Torsion free abelian groups quasiprojective over their endomorphism rings. II". . . 